\title{
Nonmodal Plasmonics: Controlling the Forced Optical Response of Nanostructures
}

\author{
Gilad Rosenblatt $\odot$, Boris Simkhovich, Guy Bartal, and Meir Orenstein \\ Department of Electrical Engineering, Technion-Israel Institute of Technology, 32000 Haifa, Israel
}

(Received 17 April 2019; revised manuscript received 25 November 2019; accepted 13 February 2020; published 25 March 2020)

\begin{abstract}
The operation of numerous physical systems and devices relies on concentrating their state space around a few carefully engineered eigenstates governing the dynamics. In photonics, these discrete degrees of freedom are typically the resonant modes of a structure. However, whenever a light source drives the structure, a continuum of additional nonmodal states generates its forced response, undermining its underlying physics and hindering control through discretization. Dealing with this nonmodal continuum poses a challenge to the design of nanophotonic systems aiming to combine compact sources and nanostructures into unified functional platforms. Here, we present a route to control forced nanostructures by engineering a discrete set of nonmodal degrees of freedom, originating from joint nanostructure-source antiresonances. We experimentally demonstrate that the forced response of ultrathin gold films is shaped by pairs of resonant-antiresonant plasmons, exhibiting joint creation and annihilation in momentum-energy space. Tuning their excitation, we show that $10 \mathrm{~nm}$ films can appear "black": exhibiting strong spectroangular wideband absorption.
\end{abstract}

DOI: 10.1103/PhysRevX.10.011071

Subject Areas: Nanophysics, Photonics, Plasmonics

\section{INTRODUCTION}

Physical interactions typically occur within a structure, designed to concentrate the continuous distribution of system states around discrete eigenstates. These discrete eigenstates constitute the principal degrees of freedom (DOFs) controlling the predominant mechanisms of interaction. Consequently, numerous physical phenomena and system designs rely on the discrete eigenstates of structures, such as discrete energy levels of quantum potentials [1], natural frequencies of mechanical oscillators [2], and discrete electromagnetic modes in photonic structures [3,4]. Reducing a continuum of system states to a few discrete levels not only dramatically scales down complexity, but also enables one to reveal the underlying physics of the system. Hence, discrete optical DOFs are frequently invoked to guide our understanding of fundamental photonic interactions, such as in determining light emission and absorption efficiencies of photonic configurations [5], information capacity of optical channels [6], performance of superresolution imaging devices [7], and even thermodynamic properties like brightness in solar concentrators [8].

A discrete eigenstate description necessitates that the modal expansion of the electromagnetic field encompasses

\footnotetext{
rosenblatt@technion.ac.il
}

Published by the American Physical Society under the terms of the Creative Commons Attribution 4.0 International license. Further distribution of this work must maintain attribution to the author(s) and the published article's title, journal citation, and DOI. the principal optical DOFs accessible in a given structure, as it does for light propagating in waveguides or trapped in cavities [3,4]. However, when a source is placed near the structure, its modes cease to provide an adequate description, as the electromagnetic wave equation becomes inhomogeneous in the region of interest [9]. Being homogeneous field solutions, the modes describe only free oscillations of light in a structure [3,9] and cannot describe the forced optical response of a structure driven by a light source. Instead, the inhomogeneous part of the field is generally attributed to a continuum of states [3,9], lacking the distinct advantage of reducing to just a few dominant optical DOFs that govern observations. For example, asymmetric line shapes in the scattering spectra of source-driven photonic structures are often phenomenologically approximated by a Fano-type interaction of a discrete mode with a continuum of scattered background radiation [10-12].

The inability of discrete modes to address the forced response associated with the transfer of energy from a driving source into the structure poses a special challenge to nanophotonic systems, where compact sources and nanostructures combine into unified platforms [13-21] to achieve nanoscale control of light-matter interactions $[22,23]$. On the one hand, nanophotonic systems frequently rely on surface plasmon polaritons (SPPs) [24]: discrete modes arising from coherent oscillations of light and charged particles at the surface of metals [25-27], twodimensional materials [28,29], metamaterials [30], transition metal nitrides [31], and other exotic optical media [32-35]. On the other hand, SPPs alone cannot capture the 
(a)

(b) $\quad d=11 \mathrm{~nm}$

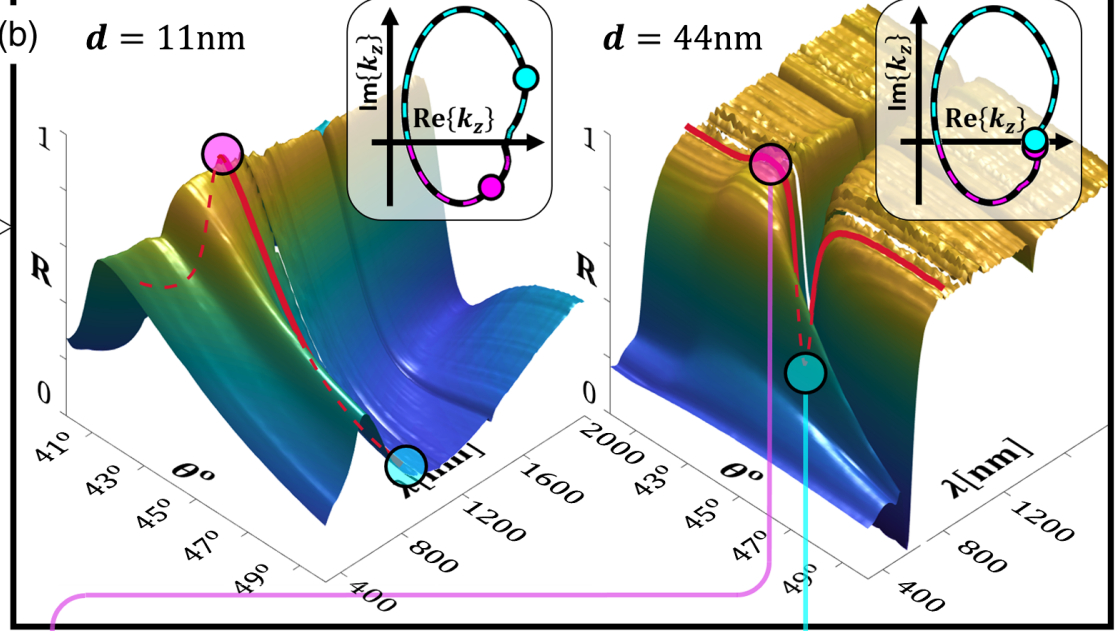

(c)

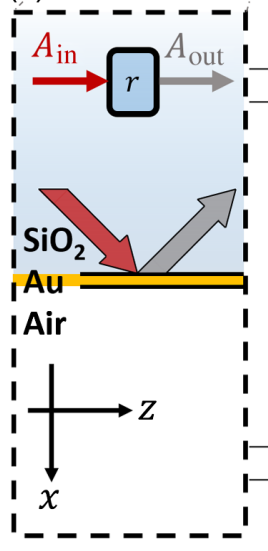

(d)
Surface plasmon polariton

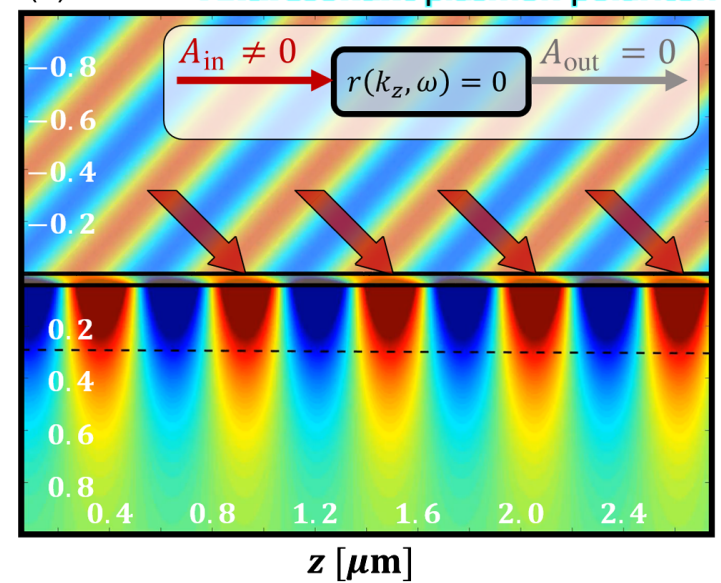

FIG. 1. Experimental concept: controlling pair excitations of modal (SPP) and nonmodal (APP) plasmons. (a) Schematic measurement setup: A TM-polarized beam impinges upon a thin gold film via a glass coupling prism, and the reflected beam is collected for various incidence angles $\theta$ and illumination wavelengths $\lambda$. (b) SPP-APP pair excitations make the measured reflectance $R$ exhibit a tunable peak-and-dip line shape $(\lambda=800 \mathrm{~nm}$, red curves), ranging from spectrally and angularly wideband absorption for ultrathin films $(11 \mathrm{~nm})$ to the narrow asymmetric Kretschmann dip for thicker films (44 nm). Inset: Locations of the APP and SPP wave vectors $k_{z}$ (cyan and magenta circles) along their root locus (dashed line) as the film thickness changes. (c) Incident $\left(A_{\text {in }}\right.$, red arrow) and reflected ( $A_{\text {out }}$, gray arrow) wave amplitudes at the front gold-glass interface are related by the reflection coefficient $r$, the transfer function of a linear space-time invariant system (block diagrams). (d),(e) Calculated magnetic fields of the SPP and APP $(d=44 \mathrm{~nm}, \lambda=600 \mathrm{~nm})$, time dynamics shown in Movie 1. Inset: SPPs and APPs assigned to system resonances $(r=\infty)$ and antiresonances $(r=0)$.

rich physics emerging in source-driven nanostructures, as nonmodal contributions are bound to be significant. Just as emission by a source is modified near nanostructures that alter the local density of states $[18,36]$, so must that source induce a tangible forced response by accessing nonmodal DOFs in those nanostructures.

Here, we show that the forced response of nanostructures originates from a discrete set of nonmodal optical DOFs that complements the discrete set of modes. We experimentally and theoretically prove the existence and prominence of nonmodal plasmonic DOFs in nanostructures, which we denote as antiresonant plasmon polaritons (APPs). Whereas SPPs are discrete resonances of free plasmonic oscillations, APPs are the discrete antiresonances emerging when those oscillations are being forced by a light source [Figs. 1(c)-1(e)]. We show that SPPs and APPs jointly shape the forced response, forming resonant-antiresonant pairs of independent plasmonic field solutions that are colocalized at a common interface, and exhibit joint "creation" and "annihilation" in momentum-energy space. With elaborate yet straightforward reflectance measurements, we unambiguously observe excitations of SPP-APP pairs in ultrathin gold films, manifested as combined peakand-dip reflectance signatures that cannot be interpreted as a Fano-type interaction of an SPP mode with a radiation continuum [Figs. 1(a) and 1(b)]. By precisely controlling 
these SPP-APP pair excitations, we demonstrate that ultrathin gold films (approximately $11 \mathrm{~nm}$ ) can appear "black": exhibiting a surprisingly strong angularly and spectrally wideband absorption (FWHM $\Delta \theta \approx 47^{\circ}, \Delta \lambda>1.34 \mu \mathrm{m}$ ). In particular, we derive design rules to engineer highly absorbing ultrathin films accurately and efficiently based on the unique properties of SPP-APP pairs.

\section{THEORY: ANTIRESONANT PLASMON POLARITONS}

Conventional theory holds that each metal-dielectric interface in a nanostructure contributes a single plasmonic DOF $[25,26,37]$. For example, a thin metal film fabricated atop a dielectric substrate supports two plasmonic modes: the short-range and long-range SPPs, respectively localized on the metal-substrate and metal-air interfaces of the film, where their field intensity is highest [Figs. 2(a) and 2(b)]. However, SPPs cannot span the entire range of plasmonic response in the nanostructure-only its homogeneous part. Most notably, they cannot describe energy transfer from an outside source into the nanostructure. This role is reserved to a discrete set of principal nonmodal plasmonic DOFs we denote as antiresonant plasmon polaritons, and the thin film supports one such APP [Fig. 2(c)].

A formal derivation of APP fields in nanostructures driven by a source, proving their independence from resonant modes, is found in Appendix A, but the main idea is the following. Assume an incident wave emitted by an outside source that impinges upon the front interface of a planar structure. The backscattering process can be described by a linear space-time invariant system, whose transfer function is the reflection coefficient $r\left(k_{z}, \omega\right)=$ $A_{\text {out }}\left(k_{z}, \omega\right) / A_{\text {in }}\left(k_{z}, \omega\right)$, and its input and output are the field amplitudes of the incident $\left(A_{\text {in }}\right)$ and reflected $\left(A_{\text {out }}\right)$ waves at the front interface for each longitudinal wave-vector component $k_{z}$ and angular frequency $\omega$ [Fig. 1(c)]. The output to any nonzero input comprises two independent parts: a homogeneous solution of the free system $\left(A_{\text {in }}=0\right)$ and a particular inhomogeneous solution of the forced system $\left(A_{\text {in }} \neq 0\right)$. The homogeneous part is spanned by the discrete modes, resonant fields obtained at singular points of the reflection $(r=\infty)$ transferring energy within or out of the structure $\left(A_{\text {in }}=0\right.$ but $\left.A_{\text {out }} \neq 0\right)$. To span the inhomogeneous part, a radiation continuum is generally invoked [3,9]. However, out of the nonmodal continuum, there is only a discrete set of field solutions that complements the modes by performing the exact opposite function: perfectly transferring energy into the structure from an outside source $\left(A_{\text {in }} \neq 0\right.$ but $\left.A_{\text {out }}=0\right)$. This discrete set of principal nonmodal DOFs consists of the discrete antiresonances shared between a structure and its driving source, when the reflection vanishes $(r=0)$ [38-40]. Consequently, the forced response of source-driven nanostructures could be primarily traced back to two discrete sets of plasmonic DOFs: resonant plasmonic modes (SPPs)
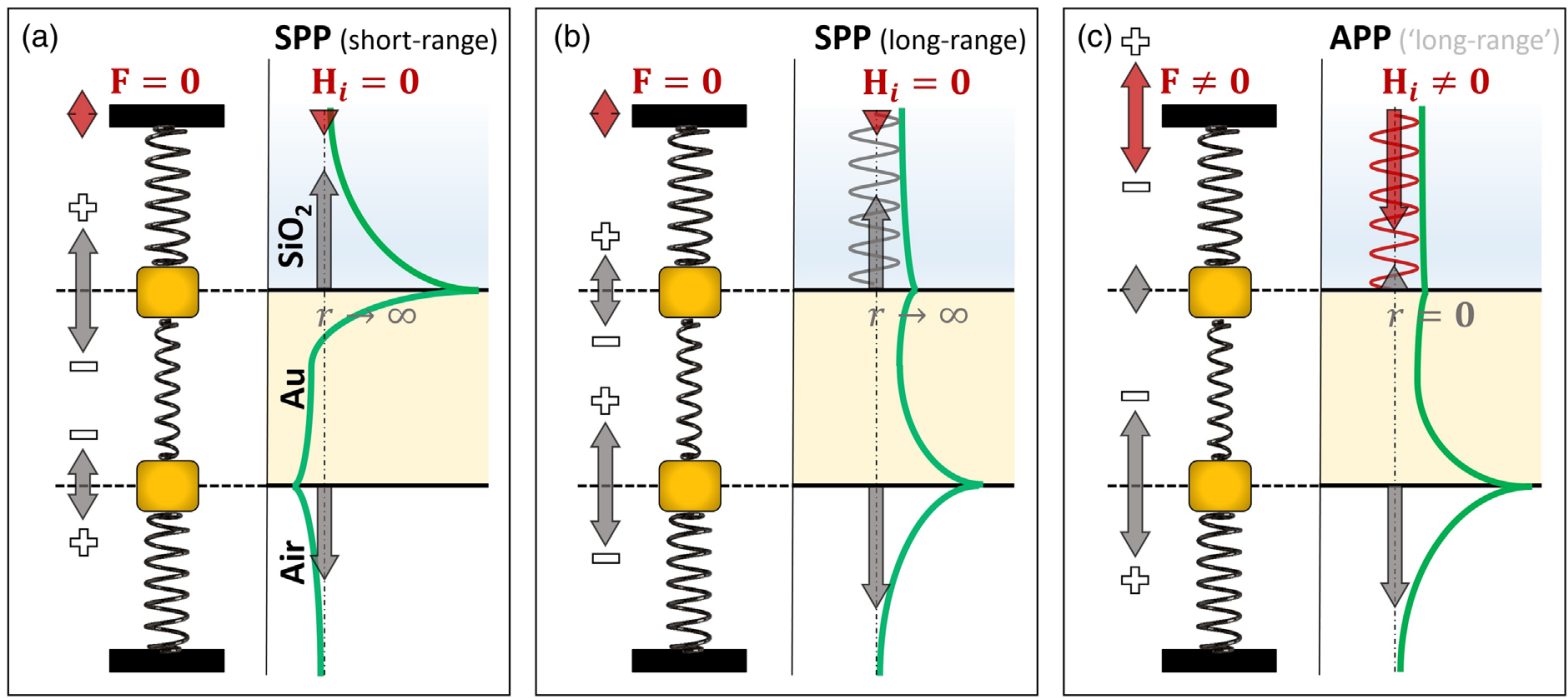

FIG. 2. Three plasmonic degrees of freedom in a thin film. Illustrated magnetic field profiles (green) of the two modal SPPs and the nonmodal APP of a thin metal film (yellow) illuminated via a dielectric substrate (light blue), and their mechanical analogue in a driven system of two coupled oscillators. (a) Short-range SPP localized on the metal-substrate interface and its anti-phase resonance analogue. (b) Long-range SPP and (c) the additional APP degree of freedom localized on the metal-air interface and their respective in-phase resonance and antiresonance analogues. Red arrows indicate the incident field amplitude $\mathbf{H}_{i}$ and driving force $\mathbf{F}$. Gray arrows indicate the scattered (reflected and transmitted) field amplitudes, and mechanical oscillation amplitudes with relative phases noted by plusminus signs. The long-range SPP comprises only a reflected field in the substrate $\left(r \rightarrow \infty, \mathbf{H}_{i}=0\right)$, whereas the APP only an incident field $\left(r=0, \mathbf{H}_{i} \neq 0\right)$. Therefore, the APP carries power into the film [red since in (c)] while the long-range SPP leaks it away [gray since in (b)], as seen in Movie 2. Illuminating from the air side leads to a different APP that is localized on the metal-substrate interface (Appendix B 7). 
that contribute the homogeneous part and antiresonant plasmonic field solutions (APPs) that contribute an inhomogeneous part [e.g., Figs. 1(d) and 1(e)].

For an intuitive explanation of the emergence of APPs, consider a simplified analogue between layered plasmonic nanostructures and systems of coupled mechanical oscillators. An isolated metal-dielectric interface functions as a plasmonic oscillator, supporting one localized resonance solution in the form of a single-interface SPP. Accordingly, a layered nanostructure acts like a system of coupled oscillators, as it produces a collective plasmonic response through evanescent coupling of adjacent metal-dielectric interfaces [41]. The principal DOFs spanning this collective response are therefore analogous to those found in a system of coupled mechanical oscillators. For instance, a thin metal film behaves as two coupled mechanical oscillators, representing its two separate metal-dielectric interfaces (Fig. 2).

If such a mechanical system is freely oscillating, its state can be fully described by a superposition of discrete collective resonances, the number of which equals the number of oscillating masses [2]. For example, a system of two oscillators supports two independent collective resonances, in which two masses coherently couple either antiphase [Fig. 2(a), left side] or in phase [Fig. 2(b), left side]. In a layered nanostructure, the analogues of these collective resonances are the collective resonant plasmonic field solutions (SPPs), the number of which equals the number of plasmonic interfaces. Consequently, the thin film supports the short-range and long-range SPPs, in which plasmonic oscillations along its two interfaces couple either antiphase [Fig. 2(a), right side] or in phase [Fig. 2(b), right side] [26].

However, when the mechanical system is driven by an external force, additional DOFs participate in its response to form the component that transfers energy into the system. A principal set of such DOFs can be described by discrete antiresonances: purely inhomogeneous solutions for which the driving force acting on the front oscillator is counterbalanced by the restoring force applied by the rest of the system, such that the front oscillator is suppressed [2]. The receptance of the front oscillator then vanishes, and so all incident power is transmitted past the driving point to the remaining oscillators. Because the front mass is idle, there are as many independent antiresonances as the number of remaining oscillating masses (see Supplemental Material [42]). For instance, two coupled oscillators support exactly one antiresonance, in which all incident power is transferred to the rear oscillator [Fig. 2(c), left side].

The plasmonic analogues of mechanical antiresonances are the discrete antiresonant field solutions we refer to as APPs. APPs can be interpreted as cases when impinging light interferes with internally reflected light to suppress plasmonic oscillations at the front interface facing the source, transferring all incident power to the remaining interfaces within the structure. Like their mechanical counterparts, there are as many independent APPs as there are plasmonic interfaces in the nanostructure, excluding the front interface. Hence, in addition to its two SPPs, the thin film supports one nonmodal plasmonic DOF in the form of an APP, which transfers incident power from a source to plasmonic oscillations at its rear interface [Fig. 2(c), right side].

In principle, therefore, each plasmonic interface in a layered nanostructure contributes two complementary DOFs: a resonant mode (SPP) intrinsic to the structure and an antiresonant field solution (APP) shared between the structure and its driving source. These resonantantiresonant pairs of plasmonic field solutions jointly shape the forced response of the nanostructure, because their fields are colocalized on their interface of origin but carry energy in opposite directions relative to the source. As we experimentally demonstrate next, the long-range SPP and APP of the thin film [Figs. 2(b) and 2(c)] form such a pair and can be controlled to allow wideband absorption by ultrathin gold films.

\section{EXPERIMENT: REFLECTANCE OF ULTRATHIN GOLD FILMS}

We experimentally prove the existence and prominence of APPs in thin metal films, since these are the simplest nanostructures to support antiresonant plasmonic field solutions. We fabricate three gold films of different thickness $(44,21$, and $11 \mathrm{~nm})$ atop fused silica right-angle prisms and measure their reflectance using a scientificgrade spectroscopic ellipsometer [Fig. 1(a)]. The measurements are taken at high angular resolution $\left(\Delta \theta=0.1^{\circ}\right.$, $40^{\circ}-50^{\circ}$ ) over a wide range of illumination wavelengths (300-2000 nm, $\Delta \lambda=10 \mathrm{~nm}$ ). A detailed description of our experimental setup and sample fabrication and characterization procedures is given in Appendix B1-B3.

The technique of exciting a plasmonic response in thin films through a coupling prism to add missing momentum to incident light was pioneered by Kretschmann and Raether [43] and is heavily relied upon in nanophotonic systems such as surface plasmon resonance biosensors [44]. However, our experiment differs from a conventional Kretschmann experiment in several key aspects. First, whereas the gold film thickness typically lies in the $50 \mathrm{~nm}$ range to obtain the sharpest reflectance dip, we employ much thinner films reaching the $10 \mathrm{~nm}$ range, where the dominant physical mechanisms affecting the response are substantially different. Second, we fabricate those films atop glass substrates using a one-molecule-thick adhesion layer, instead of standard bulkier lossy adhesion layers such as $\mathrm{Ti}$ or $\mathrm{Cr}$ that affect the optical properties of the sample (monolayer characterization presented in Appendix B 2). This procedure ensures our measurements faithfully represent the response of ultrathin gold films, and 

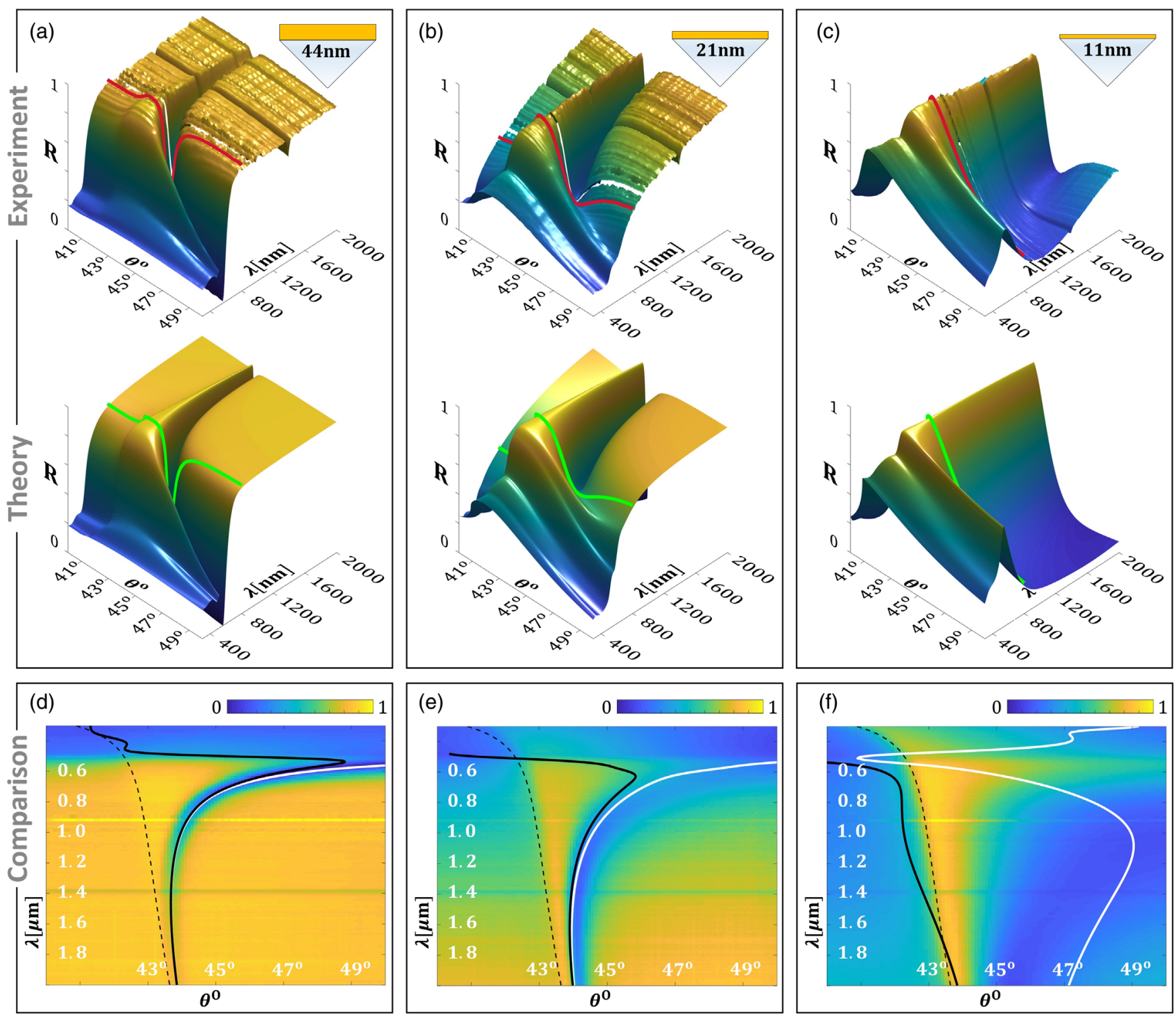

FIG. 3. Experimental results: excitations of APP and SPP degrees of freedom in thin gold films. (a)-(c) Measured (top) and predicted (bottom) reflectance from a (a) 44, (b) 21 , and (c) $11 \mathrm{~nm}$ thin gold film fabricated atop a glass coupling prism as a function of incidence angle $\theta$ and illumination wavelength $\lambda$, also shown in Movie 3. (d)-(f) Calculated real part of the long-range SPP (black) and APP (white) dispersion curves overlaid atop the measured reflectance for each film. Dashed black lines denote the critical angle. Red and green curves in (a)-(c) highlight the measured and predicted reflectance at $\lambda=800 \mathrm{~nm}$ analyzed in Fig. 4 . The offset between regions of high and low reflectance and the black and white curves in (f) is explained in Appendix B 8.

the effects we measure are not artifacts of an additional layer. Additionally, we can trace the dispersion of observed features, because we acquire angular line shapes over a wide spectral range.

Our reflectance measurements are presented in Figs. 3(a)-3(c) as a function of the incidence angle $(\theta)$ and illumination wavelength $(\lambda)$ and are all in excellent agreement with the theoretical prediction (derived in Appendix B 5). The measurements show a qualitative change in the response as the film thickness decreases. The thickest sample [Fig. 3(a)] exhibits the typical signature of a Kretschmann experiment at $\lambda=600 \mathrm{~nm}$
[Fig. 1(b), red curve]: a pronounced zero-reflectance dip at an above-critical angle over a total internal reflection background, known as the Kretschmann dip [26,27,43]. However, the two thinner samples [Figs. 3(b) and 3(c)] exhibit utterly different responses that feature both a peak and a dip, implying that more than a single plasmonic DOF is at play. In particular, the response of the ultrathin gold film [Fig. 3(c)] is the one most central to this work: Instead of approaching the response of a bare glass prism for such a vanishingly thin film $(11 \mathrm{~nm})$, which above the critical angle is total internal reflection, we observe strong absorption over wide angular and spectral ranges, with estimated 
FWHM bandwidths of $\Delta \theta \approx 47^{\circ}$ and $\Delta \lambda>1.34 \mu \mathrm{m}$ (see Fig. 7).

Before uncovering the physical mechanism enabling ultrathin gold films to appear black, we first identify which plasmonic DOFs dominate their forced response. We trace back the prominent peak and dip features in the reflectance to the two discreet plasmonic DOFs accessible from the far field using the coupling prism: the resonant long-range SPP [Fig. 2(b)] and the antiresonant APP [Fig. 2(c)], colocalized on the rear gold-air interface of the gold film. In Figs. 3(d)3(f), we compare the real part of their calculated dispersion curves (derived in Appendix B 4) with the measured reflectance signatures.

For the thickest sample [44 nm, Fig. 3(d)], the longrange SPP dispersion curve (black) seems to fit the measured reflectance dip angle over most wavelengths. This fit is partly why the standard theory associates the formation of the Kretschmann dip line shape with an SPP excitation [25-27,43], despite competing explanations [45-49]. Recently, the asymmetry of the Kretschmann dip line shape was attributed to Fano-type interference of the SPP with a continuum of scattered background radiation [50], which can account for small displacements in the dip angle [51]. However, our measurements reveal a significant discrepancy between the Kretschmann dip angle and the long-range SPP dispersion curve near the surface plasma frequency $(\lambda \approx 530 \mathrm{~nm})$, where the long-range SPP dispersion undergoes backbending while the measured dip feature does not. This empirical discrepancy has recently posed a challenge to the standard theory [52]. Nevertheless, it does not yet disprove it, because near the backbending the above comparison becomes problematic: The long-range SPP wave vector attains a large imaginary part, so its dispersion is no longer faithfully represented solely by the real part.

Remarkably, at the same film thickness range, the nonmodal APP dispersion curve (white) fits the measured dip angle extremely well throughout the entire range of measurement [44 nm, Fig. 3(d)]. It does not undergo the same type of backbending and, instead, splits apart from the long-range SPP dispersion curve. To definitively attribute the reflectance dip feature to an APP excitation rather than an SPP, this splitting must be demonstrated away from the long-range SPP backbending region. To that end, we explore thinner gold films, where we observe two wellseparated regions of high and low reflectance, a pronounced peak and a dip. These two regions strongly split apart as the film thickness decreases once from 44 to $21 \mathrm{~nm}$ [Fig. 3(e)] and again to $11 \mathrm{~nm}$ [Fig. 3(f)], marking a clear trend. Both this splitting and its trend coincide with those of the strong splitting between the predicted long-range SPP and APP dispersion curves across the entire 500-2000 nm spectral range. Hence, our experiment confirms that the forced plasmonic response of thin gold films can be traced back to two principal DOFs, the long-range SPP and APP:
The APP is most strongly excited at the reflectance dip, whereas the long-range SPP at the reflectance peaksubstantially departing from the prevailing theory of the past five decades.

We emphasize that the ultrathin film reflectance signatures we measure cannot be explained by Fano models, based on interference of a discrete SPP mode and a continuum of scattered background radiation (see Appendix C). From a quantitative standpoint, Fano line shapes simply do not fit any of the particular peak-and-dip line shapes emerging when the gold film is thinner than $25 \mathrm{~nm}$ (Figs. 9 and 10). Qualitatively, the Fano model describes a fundamentally different mechanism to that which dominates the forced response of ultrathin gold films. Rather than being formed by weak coupling of a discrete state (narrow) and a continuum (broad) [10], the forced response of ultrathin films emerges from two discrete states that are entirely uncoupled and have comparable widths. Hence, while a Fano line shape can phenomenologically approximate the asymmetric zeroreflectance Kretschmann dip for thicker films [50,51], it cannot predict nor explain the wideband absorption we observe for ultrathin gold films.

\section{WIDEBAND ABSORPTION BY ULTRATHIN GOLD FILMS}

To unravel the physical mechanism making ultrathin gold films appear black, we track the two principal modal and nonmodal DOFs shaping the forced response in the reflection analysis depicted in Fig. 4. The measured angular reflectance signatures [Figs. 4(a)-4(d), fitting colored lines in Figs. 3(a)-3(c)] describe the thin film response only over real-valued wave vectors. Capturing the entire system response requires calculating the reflection coefficient $r$ over the complex wave-vector domain $k_{z}$ [Figs. 4(e)-4(h)]. This representation highlights the two principal DOFs, the long-range SPP and APP, appearing as reflection pole (magenta) and zero (cyan) points, respectively. The measured reflectance at each incidence angle [Figs. 4(b)-4(d), red] is determined by $|r|^{2}$ at a respective point along the real $k_{z}$ axis [Figs. 4(a)-4(d), green] (see Appendix B 4). Hence, we retrieve the reflectance of each sample by tracking how the pole and zero project onto the real axis [Figs. 4(e)-4(h), dashed black line], following equal- $|r|$ contour lines. Evidently, these projections imprint a peak-and-dip reflectance signature (black and white arrows). However, this signature is strongly affected by the particular thicknessdependent pole and zero locations, which draw a peculiar trajectory in the complex domain.

Because the long-range SPP and APP are colocalized at the rear interface, their pole-zero trajectory obeys a pairwise symmetry, forming the close-loop root locus depicted in Fig. 4 insets (shown in detail in Fig. 7 and Movie 4). At the asymptotic ends of this root locus, the pole and zero are jointly created and annihilated: As the film thickness 

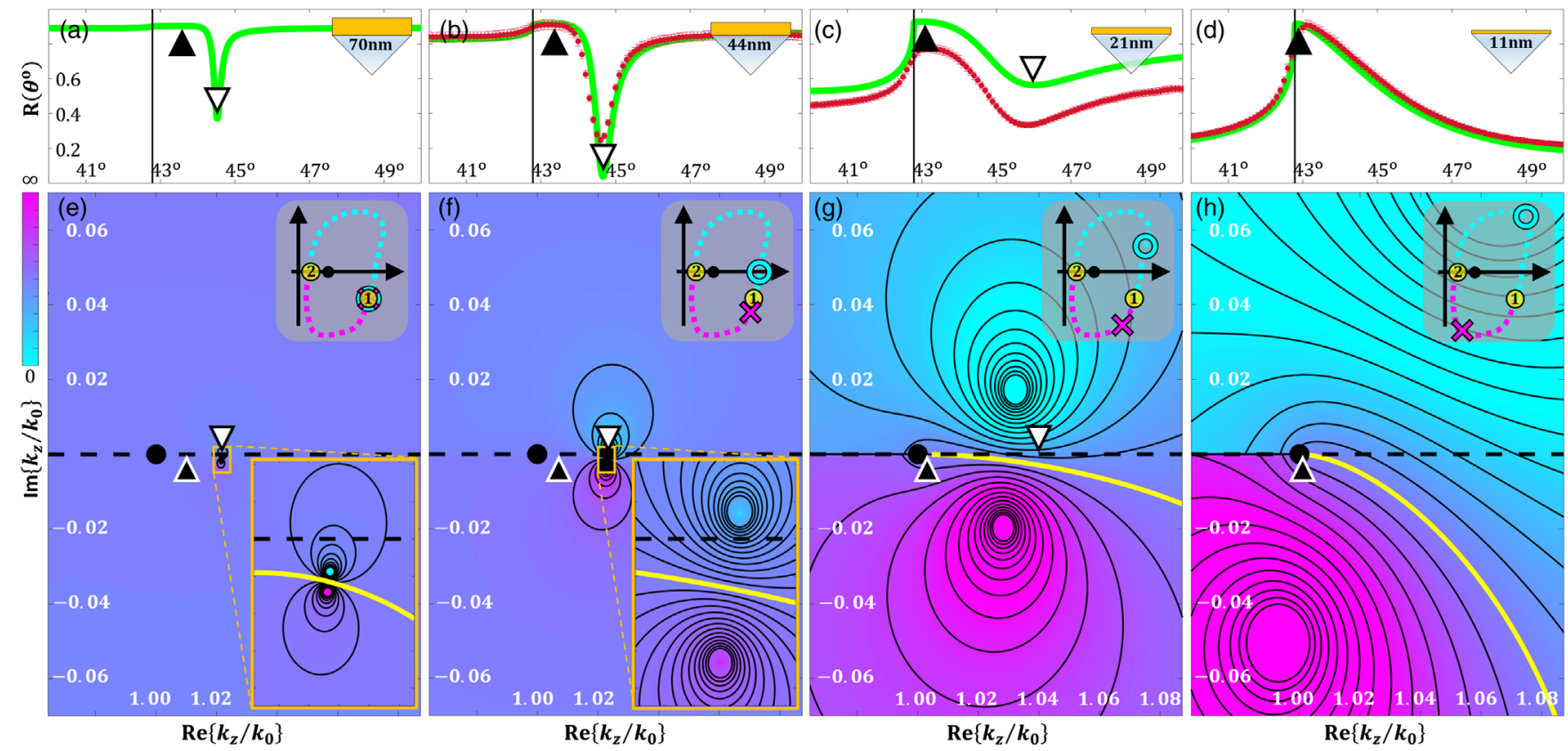

FIG. 4. Pairing of nonmodal (APP) and modal (SPP) plasmonic DOFs. (a)-(d) Measured (red) and predicted (green) reflectance of (a) 70, (b) 44, (c) 21, and (d) $11 \mathrm{~nm}$ gold films illuminated through a glass coupling prism as a function of incidence angle $\theta$ at illumination wavelength $\lambda=800 \mathrm{~nm}$. (e)-(h) Calculated reflection coefficient magnitude $|r|$ of the respective films (log scale) for complex longitudinal wave vectors $k_{z}$ corresponding to the angular range in (a)-(d). Enlarged sections marked by orange rectangles. The long-range SPP and APP correspond to reflection pole (magenta) and zero (cyan) points, respectively. Constant $|r|$ contour lines illustrate how the pole-zero pair projects onto the real axis (dashed black line) a reflectance peak-and-dip signature (black and white arrows). The angular shift between these two features arises from the tilt highlighted by a downward sloping $|r|=1$ line (yellow). Inset: Pole-zero locations along their joint closed-loop root locus as the film thickness decreases from $\infty \rightarrow 0$ (points $1 \rightarrow 2$ ), shown through the entire progression in Movie 4. Vertical black lines in (a)-(d) and black dots in (e)-(h) mark the critical angle $\left(k_{z}=k_{0}\right)$.

exceeds the penetration depth of light into the gold film, the rear gold-air interface becomes inaccessible, and the pole and zero recombine and cancel (yellow point 1). Con versely, as the film thickness vanishes entirely, the pole and zero coalesce at the Brewster angle of the emerging glassair interface (yellow point 2) (see Appendix B 6).

Traveling in between those ends, the pole and zero locations are bound by the local energy balance at the rear interface: The APP pumps energy into that interface, while the long-range SPP leaks it away. Consequently, any farfield source excites the APP more than the long-range SPP to maintain a net energy influx, and so the associated polezero pair is tilted in a way that ensures a smaller-than-one reflectance above the critical angle (the yellow $|r|=1$ line stays under the real axis; see Supplemental Material [42]). When this tilted pair projects onto the real axis, it imprints the reflectance peak-and-dip signatures we experimentally observe. The dip indicates a stronger energy transfer from the source to plasmonic oscillations via the APP excitation, whereas the peak indicates a reduction in plasmonic energy consumption due to an increased long-range SPP excitation, as it destructively interferes with the APP at the rear interface leading to higher reflectance.

The pole and zero root loci are divided into three regions, in between which the peak-and-dip signature strongly varies. First, once the film becomes sufficiently thin to facilitate coupling between its two interfaces, the pole and zero split apart: The zero draws closer to the real axis, whereas the pole moves further away $[70 \mathrm{~nm}$, Fig. 4(e)]. Because the zero is closer, it projects more strongly onto the real axis than the pole, leading to a pronounced reflectance dip preceded by a subtler reflectance peak closer to the critical angle [Fig. 4(a)]. Second, as the film thickness further decreases below a critical value, the zero crosses the real axis, and the pole and zero now lie on opposite sides [21 nm, Fig. 4(g)]. Therefore, both strongly project onto the real axis, giving rise to pronounced peak and dip features [Fig. 4(c)]. Finally, as the film gradually vanishes, splitting between the pole and zero eventually stops as they converge towards their coalescence point, retrieving the flat total internal reflection response of a bare glass prism (see Movie 4).

Each transition between different regions of the pole and zero root loci marks a distinct physical phenomenon. For example, the first transition takes place as the zero crosses the real axis [44 nm, Fig. 4(f)]. Here, the energy the APP pumps into the thin film exactly compensates for its plasma loss, so it exhibits no net propagation loss, attaining a purely real wave vector. Consequently, the APP becomes directly and fully excitable from the far field, 
imprinting the well-known zero-reflectance Kretschmann dip [Fig. 4(b)].

However, it is the second transition that leads to wideband absorption by ultrathin gold films [11 nm, Fig. 3(c)]. It occurs at the ultrathin thickness range, in which the longrange SPP (pole) and APP (zero) are maximally split apart in momentum [Fig. 4(h); the exact design obeys Eqs. (B9)-(B12) in Appendix B 9], before rapidly coalescing at the vanishingly thin film limit (see Movie 4). Precisely in this narrow thickness range, the tilted polezero pair projects the widest most separated signatures onto the real axis. However, whereas the wide projection of the zero can be observed by far-field measurements over the entire angular range, that of the pole is observable only above the critical angle, cutting the peak short due to the influence of the light cone branch point in the topologically nontrivial momentum space (see Appendix B 6, Fig. 7, Movie 4). Consequently, the maximally split pole-zero pair imprints an atypical signature: a narrow reflectance peak squeezed onto the critical angle, followed by an angularly wide absorption dip with its center pushed to higher angles [Figs. 3(c), 4(d), 7(c), and 7(f)]. In principle, this angularly wideband absorption spans the entire spectral range at which gold has a plasmonic response, because the closeloop trajectory of the long-range SPP and APP pair in momentum space is topologically guaranteed at each such wavelength. It is therefore the maximally split state of a resonant-antiresonant plasmon pair that enables the wideband absorption we measure for the $11 \mathrm{~nm}$ ultrathin gold film [Fig. 3(c)], with an estimated spectroangular FWHM bandwidth of $\Delta \theta \approx 47^{\circ}$ and $\Delta \lambda>1.34 \mu \mathrm{m}$ (Fig. 7). Detailed design rules for this black ultrathin film effect based on properties of the long-range SPP-APP root locus are given in Appendix B9.

In our experiment, we use film thickness as a tuning parameter to control the optical response by moving the long-range SPP and APP pair to desired locations along a closed-loop trajectory. Generally, any parameter that regulates coupling to the interface on which a target SPP-APP pair is localized can be used to control its contribution to the line shape. Each such parameter generates its own closed-loop trajectory for the pair. Hence, while any single tuning parameter affects both the SPP and APP of a pair, combining different tuning parameters can allow separate control of the two.

Finally, we note that the APPs a structure supports depend on the direction from which it is illuminated. Hence, illuminating the gold film from the air rather than glass side leads to a different APP whose field is localized on the gold-glass interface, and it follows a closed-loop trajectory with the short-range SPP (Appendix B 7).

\section{DISCUSSION AND SUMMARY}

In this work, we propose and implement the concept of nonmodal plasmonics, rooted in the fact that light sources and nanostructures actively share a principal set of discrete optical degrees of freedom. We discover a new class of plasmonic DOFs, identified not with the modes of photonic nanostructures but with the discrete antiresonances that form when those nanostructures are being driven by a light source. These antiresonant plasmon polaritons transfer energy from the source into the nanostructure, playing a pivotal role in shaping its forced optical response. APPs coexist with their modal SPP counterparts, which scatter energy away from or within the nanostructure. Therefore, the interference of APPs and SPPs regulates the energy influx entering the nanostructure from the source, although APPs also support pure and direct excitation when they exactly compensate for plasma loss.

Measuring the spectroangular reflectance of ultrathin gold films, we unambiguously demonstrate that APPs and SPPs are jointly accessible to external excitation, manifested as reflectance dip and peak signatures. Albeit having utterly different dispersions, SPPs and APPs form resonant-antiresonant pairs that are colocalized at a common interface and exhibit joint creation and annihilation in momentum-energy space. We experimentally demonstrate precise control of such SPP-APP pairs and show it can dramatically alter the linear optical response of passive structures: enabling strong spectrally and angularly wideband absorption for ultrathin gold films $(11 \mathrm{~nm})$, with estimated FWHM bandwidths of $\Delta \theta \approx 47^{\circ}$ and $\Delta \lambda>1.34 \mu \mathrm{m}$. In particular, we derive design rules to engineer such a black film response based solely on properties of SPP-APP pairs. Similarly, intricate forced responses can be designed by controlling multiple SPPAPP pairs at different interfaces of a nanostructure, each designed to govern a separate angular range.

APPs break the long-standing paradigm that each plasmonic interface contributes a single DOF, supporting instead a pair of modal and nonmodal DOFs. Furthermore, by reducing the continuum of nonmodal system states to a few principal discrete solutions, APPs join with SPPs to provide a purely discrete description of the forced response in source-driven nanostructures. Hence, APPs add key channels that can greatly benefit the control of light-matter interactions in nanostructures and open up fresh avenues for nanophotonics research and application. In particular, APPs can be leveraged to mold the absorption spectra of photonic nanostructures to achieve high-efficiency plasmon-assisted light harvesting or enhanced plasmonic sensing in future nanophotonic devices.

\section{ACKNOWLEDGMENTS}

We gratefully acknowledge expert advice on ellipsometry by Guy Ankonina, assistance in sample preparation by Shimon Dolev, and meticulous proofreading by Grisha Spektor. Measurements and fabrication were done at the Technion Photovoltaic Laboratory in the MNFU clean room facilities. This research was partially supported by the Israeli Centers of Research Excellence "Circle of 
Light.” G. R., B. S., and M. O. gratefully acknowledge funding from the Israeli Innovation Authority, programs 61938 and 65616.

\section{APPENDIX A: ANTIRESONANT DEGREES OF FREEDOM IN THE FORCED OPTICAL RESPONSE}

Antiresonant field solutions are zeros in the field amplitude reflection coefficient of a structure, denoting discrete wave vectors at which the source and structure are impedance matched. Hence, their field distribution incorporates an incoming wave that transfers all incident power into the structure, without reflecting an outgoing wave back toward the source [Fig. 2(c)]. In contrast, resonant modes are discrete reflection poles whose field includes an outgoing wave directed away from the structure, but no incoming wave [Figs. 2(a) and 2(b)]. In this appendix, we show that antiresonant field solutions are linearly independent from resonant modes.

\section{Mapping the backscattering process to a scalar linear system}

Assume that a driving light source emits a polarized wave that propagates in a homogeneous substrate $(x \leq 0)$ and is subsequently backscattered from the front interface of a planar structure $(x=0)$. We assume TM polarization and choose a coordinate system such that the surface normal and magnetic fields are directed along the $\mathbf{x}$ and $\mathbf{y}$ axes, respectively [Figs. 1(a) and 1(c); for TE polarization, similar equations hold for the electric field]. The magnetic fields of the incident $\left(\mathbf{H}_{\text {inc }}\right)$ and reflected $\left(\mathbf{H}_{\text {ref }}\right)$ waves can therefore be spectrally decomposed in the temporal $(t)$ and spatial $(z)$ domains and expressed in terms of scalar complex field amplitude functions $A_{\text {in }}$ and $A_{\text {out }}$ :

$$
\begin{aligned}
& \mathbf{H}_{\mathrm{inc}}(x \leq 0, z, t)=\mathbf{y} \int_{-\infty}^{\infty} \int_{-\infty}^{\infty} A_{\mathrm{in}}\left(k_{z}, \omega\right) e^{j\left(\omega t-k_{z} z\right)} e^{-j k_{x} x} d k_{z} d \omega, \\
& \mathbf{H}_{\mathrm{ref}}(x \leq 0, z, t)=\mathbf{y} \int_{-\infty}^{\infty} \int_{-\infty}^{\infty} A_{\text {out }}\left(k_{z}, \omega\right) e^{j\left(\omega t-k_{z} z\right)} e^{+j k_{x} x} d k_{z} d \omega,
\end{aligned}
$$

where $\mathbf{y}$ is a unit vector along $y, k_{z}$ and $k_{x}$ are, respectively, the parallel $(z)$ and normal $(x)$ wave-vector components to the front interface, satisfying a quadratic relation $k_{x}^{2}+k_{z}^{2}=$ $(\omega / c)^{2} \varepsilon \mu$ imposed by the wave equation, $\varepsilon$ and $\mu$ are the permittivity and permeability, respectively, of the substrate, $\omega$ is the angular frequency, and $c$ is the speed of light in vacuum. Because the space-time domain fields are real valued, the scalar complex field amplitudes satisfy $A_{\text {in }}\left(k_{z}, \omega\right)=A_{\text {in }}^{*}\left(-k_{z},-\omega\right)$ and $A_{\text {out }}\left(k_{z}, \omega\right)=A_{\text {out }}^{*}\left(-k_{z},-\omega\right)$, and the $k_{x}$ root branch selection obeys $k_{x}\left(k_{z}, \omega\right)=$ $-k_{x}^{*}\left(-k_{z},-\omega\right)$. Furthermore, to ensure that the incident and reflected waves carry energy into and away from the structure, respectively, the integration is performed over the $\operatorname{Im} k_{x}<0$ root branch, corresponding to the proper Riemann sheet in the Sommerfeld branch cut selection [9].

The interaction between the incident wave and the structure is governed by Maxwell's equations and boundary conditions for the fields throughout the structure. These are all encapsulated in the field amplitude reflection coefficient $r$, relating the field amplitudes of incident and reflected plane waves at the interface facing the source:

$$
r\left(k_{z}, \omega\right)=A_{\text {out }}\left(k_{z}, \omega\right) / A_{\text {in }}\left(k_{z}, \omega\right) .
$$

Therefore, the process of backscattering by a planar structure can be described by a linear system whose scalar input and output are the complex field amplitude functions $A_{\text {in }}$ and $A_{\text {out }}$, respectively [Fig. 1(c), inset]. This system is both time invariant and translation invariant in the $z$ direction, and its temporal $(\omega)$ and spatial $\left(k_{z}\right)$ transfer function is the field amplitude reflection coefficient $r\left(k_{z}, \omega\right)$.

\section{Mapping the linear system output to homogeneous and inhomogeneous field components}

As a linear system, the output amplitude $A_{\text {out }}$ for any nonzero input amplitude $A_{\text {in }}$ comprises two contributions, jointly satisfying appropriate initial conditions in $z$ and $t$ :

$$
A_{\text {out }}=A_{H}+A_{I},
$$

a homogeneous part $A_{H}$ solving the free system $\left(A_{\text {in }}=0\right)$, and a particular inhomogeneous part $A_{I}$ solving the forced system $\left(A_{\text {in }} \neq 0\right)$. The inhomogeneous part is unique up to a homogeneous solution but cannot be described solely in terms of homogeneous solutions.

Hence, the total forced field solution in the substrate $\mathbf{H}_{F}=\mathbf{H}_{\text {inc }}+\mathbf{H}_{\text {ref }}$ can be decomposed into what we denote as homogeneous $\left(\mathbf{H}_{H}\right)$ and inhomogeneous $\left(\mathbf{H}_{I}\right)$ components, associated with the homogeneous and inhomogeneous parts of the system response given by Eq. (A4):

$$
\begin{aligned}
\mathbf{H}_{H}(x \leq 0, z, t)= & \mathbf{y} \int_{-\infty}^{\infty} \int_{-\infty}^{\infty} A_{H}\left(k_{z}, \omega\right) e^{+j k_{x} x} e^{j\left(\omega t-k_{z} z\right)} d k_{z} d \omega, \\
\mathbf{H}_{I}(x \leq 0, z, t)= & \mathbf{y} \int_{-\infty}^{\infty} \int_{-\infty}^{\infty} A_{\text {in }}\left(k_{z}, \omega\right) e^{-j k_{x} x} e^{j\left(\omega t-k_{z} z\right)} d k_{z} d \omega \\
& +\mathbf{y} \int_{-\infty}^{\infty} \int_{-\infty}^{\infty} A_{I}\left(k_{z}, \omega\right) e^{+j k_{x} x} e^{j\left(\omega t-k_{z} z\right)} d k_{z} d \omega,
\end{aligned}
$$


where the inhomogeneous field component consists of the driving field in addition to the inhomogeneous part of the reflected field.

\section{Linear independence of the discrete resonant and antiresonant field solutions}

The homogeneous field component $\mathbf{H}_{H}$ is spanned by the discrete optical degrees of freedom that are intrinsic to the structure, given by the set of resonant electromagnetic modes $\left(A_{\text {in }}=0\right.$ but $\left.A_{\text {out }} \neq 0\right)$

$$
\begin{aligned}
A_{H}= & \sum_{m} \frac{a_{m}}{2} \delta\left(k_{z}-k_{z, R_{m}}\right) \delta\left(\omega-\omega_{R_{m}}\right) \\
& +\sum_{m} \frac{a_{m}^{*}}{2} \delta\left(k_{z}+k_{z, R_{m}}\right) \delta\left(\omega+\omega_{R_{m}}\right),
\end{aligned}
$$

where $a_{m}$ is the complex amplitude of the $m$ th mode in the expansion. This result is because $A_{H}$ satisfies Eqs. (A3) and (A4) for $A_{\text {in }}=A_{I}=0$ and, therefore, consists only of discrete spatial $\left(k_{z, R m}\right)$ and temporal $\left(\omega_{R m}\right)$ frequency contributions that satisfy the resonance condition of the structure

$$
r\left(k_{z, R_{m}}, \omega_{R_{m}}\right)=\infty,
$$

which is by definition the dispersion relation of resonant modes [3,9]. In particular, $\mathbf{H}_{H}$ lacks an incident wave component in the substrate that could laterally transfer energy from the driving source into the structure and transfers energy only within or out of the structure.

To account for the inhomogeneous field component $\mathbf{H}_{I}$, additional nonmodal degrees of freedom are required, and those are generally described by a continuum of radiation states (e.g., using a Green's function formalism) [3,9,53]. Yet out of this nonmodal continuum, there is only a discrete subset of field solutions that complements the modes by performing their exact opposite function: transferring all energy from an outside source into the structure $\left(A_{\text {in }} \neq 0\right.$ but $A_{\text {out }}=0$ ). Its contribution to $\mathbf{H}_{I}$ can thus be encapsulated into the driving field

$$
\begin{aligned}
\tilde{A}_{\text {in }} \triangleq & \sum_{m} \frac{b_{m}}{2} \delta\left(k_{z}-k_{z, A_{m}}\right) \delta\left(\omega-\omega_{A_{m}}\right) \\
& +\sum_{m} \frac{b_{m}^{*}}{2} \delta\left(k_{z}+k_{z, A_{m}}\right) \delta\left(\omega+\omega_{A_{m}}\right),
\end{aligned}
$$

over the discrete spatial $\left(k_{z, A m}\right)$ and temporal $\left(\omega_{A m}\right)$ frequencies that satisfy the antiresonance condition shared between the structure and its driving source (perfect impedance matching)

$$
r\left(k_{z, A_{m}}, \omega_{A_{m}}\right)=0,
$$

where $b_{m}$ is the amplitude of the $m$ th antiresonant field solution.

The forced field solution $\mathbf{H}_{F}$ can therefore be expanded into three distinct contributions by discrete modes $\left(\mathbf{H}_{H}\right)$, discrete antiresonant solutions $\left(\mathbf{H}_{I, D}\right)$, and a remaining continuum of radiation states $\left(\mathbf{H}_{I, C}\right)$ :

$\mathbf{H}_{H}(x \leq 0, z, t)=\mathbf{y} \sum_{m} \operatorname{Re}\left\{a_{m} e^{+j k_{x, R_{m}} x} e^{j\left(\omega_{z, R_{m}} t-k_{z, R_{m}} z\right)}\right\}$,

$$
\begin{gathered}
\mathbf{H}_{I, D}(x \leq 0, z, t)=\mathbf{y} \sum_{m} \operatorname{Re}\left\{b_{m} e^{-j k_{x, A_{m}} x} e^{j\left(\omega_{z, A_{m}} t-k_{z, A_{m}} z\right)}\right\}, \\
\mathbf{H}_{I, C}=\mathbf{H}_{I}-\mathbf{H}_{I, D} .
\end{gathered}
$$

The independence of the resonant $\left(\mathbf{H}_{H}\right)$ and antiresonant $\left(\mathbf{H}_{I, D}\right)$ field contributions stems from the linear independence of the homogeneous $\left(A_{H}\right)$ and inhomogeneous $\left(A_{I}\right)$ parts of the linear system response $A_{\text {out }}$. Namely, for the case of an antiresonance field solution $\left(A_{\text {out }}=0\right)$, both $A_{H}$ and $A_{I}$ independently vanish, so its homogeneous field component $\mathbf{H}_{H}$ contributed by resonant modes (derived from $A_{H}$ ) must vanish. Consequently, resonant modes do not contribute to antiresonant field solutions (and vice versa) - and, in particular, these two distinct types of field solutions are linearly independent.

\section{APPENDIX B: METHODS}

This appendix covers all details related to the experimental setup, sample preparation and characterization, and data analysis.

\section{Experimental setup}

Our measurement setup is schematically illustrated in Fig. 1(a). It consists of a J.A. Woollam variable angle spectroscopic ellipsometer (VASE) operated in RT data acquisition mode using focusing lenses. The light source is a wideband xenon lamp, which is filtered by a monochromator to a spectral width of about 5-10 nm (depending on the automatically set monochromator exit slit width), coupled into an optical fiber, and collimated at the fiber tip so that the emerging beam is roughly $3 \mathrm{~mm}$ in diameter. The emerging beam is then TM polarized and propagates in free space until it is focused by a lens to roughly $0.7 \mathrm{~mm}$ in diameter, when it impinges on the coupling prism.

The sample is mounted on a rotation stage, such that the specular reflection is collected by a correspondingly rotating detection arm. The collected signal is integrated over rotating analyzer orientations to produce a reflectance measurement at each incidence angle and illumination wavelength. Measurements are taken for incidence angles between $40^{\circ}$ and $50^{\circ}$ at a $\Delta \theta=0.1^{\circ}$ step size and 
illumination wavelengths between 300 and $2000 \mathrm{~nm}$ at a $\Delta \lambda=10 \mathrm{~nm}$ step size.

The spectral range of measurement is limited by the transmission band of the optical fiber, whereas the angular range is limited by a systemic offset error caused by refraction inside the coupling prism. The beam coming out of the prism is laterally shifted compared to the hypothetical beam that would have been directly reflected from a thin flat target, which the detection arm is designed to collect (Fig. 6). This lateral shift produces a signal drop if

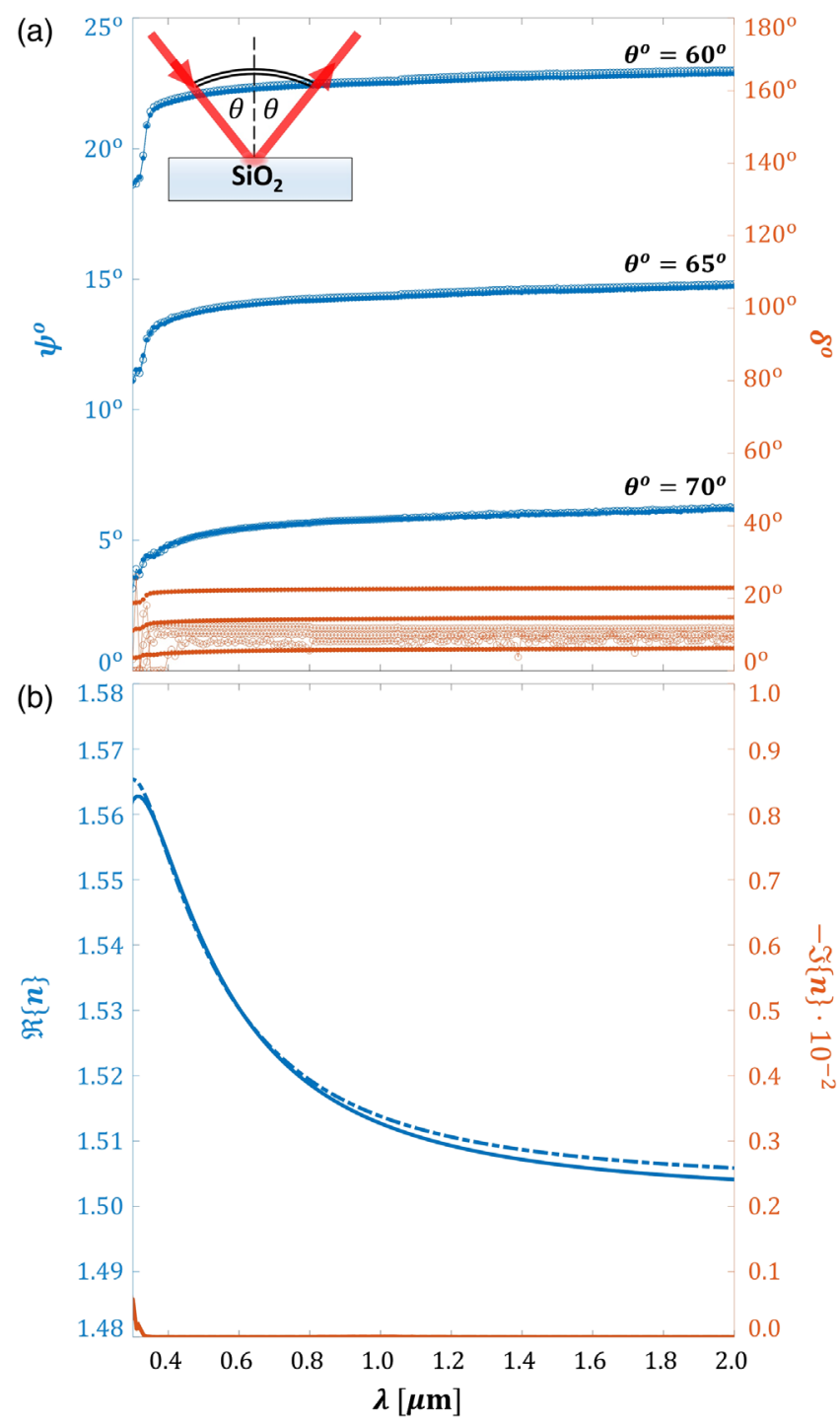

FIG. 5. Ellipsometry of chemically treated glass substrates. (a) Measured ellipsometry parameters $\psi$ (blue, left axis) and $\delta$ (orange, right axis), for treated (hollow circles) and nontreated (dots) cover glass substrates as a function of illumination wavelength $\lambda$ at three incidence angles $\theta$ (noted inset). (b) Real (blue, left axis) and imaginary (orange, right axis) parts of the refractive index $n$ of the treated (dashed line) and nontreated (solid line) glass covers fitted from the measured data in (a). The change in $n$ is smaller than $2.5 \times 10^{-3}$ over the $300 \mathrm{~nm}-2 \mu \mathrm{m}$ spectral range. the reflected beam partially overlaps with the detector instead of being fully collected. The angular range for full collection is defined by the condition that the angledependent lateral shift plus the beam radius be smaller than half the detector length dimension. For right-angle glass coupling prisms, this angular range is roughly $\pm 8^{\circ}$ around $\theta=45^{\circ}$.

\section{Sample preparation}

We prepare three samples consisting of a thin gold film fabricated atop a right-angle $10 \mathrm{~mm}$ fused silica prism using thermal evaporation in three separate sessions. Typically, evaporation of gold over glass substrates requires an adhesion layer several nanometers thick of a transition metal such as Ti or Cr. However, we avoid the use of such bulky and lossy adhesion layers, as their material loss alters the optical properties of the fabricated sample and they act as an additional layer that would add coherent effects associated with three-layer structures. While these effects may be negligible for $50 \mathrm{~nm}$ gold film samples, they become significant for ultrathin gold films such as our 11-nm-thick gold film.

Instead, we follow the process described in Ref. [54]. We treat the prism surface with (3-Mercaptopropy1)trimethoxysilan to create a molecular coating of an insulating organic coupling agent that acts as a single-atom-thick adhesive. As we experimentally verify for our films, this monolayer does not significantly affect the optical properties of the sample [54]. Measuring the ellipsometric parameters of glass cover slides (Schott D263) before and after treatment [Fig. 5(a)] and fitting the measured data using a Cauchy model [Fig. 5(b)], we observe no significant change in the refractive index $n$ of the glass substrates $\left(|\Delta n| \leq 2.5 \times 10^{-3}\right)$. This procedure ensures that the signatures we measure arise from the ultrathin gold film and are not artifacts of an additional layer.

\section{Sample characterization}

Alongside each of the three prism samples, we fabricate control samples on flat glass cover slides and silicon wafers. These are used to measure the gold film thickness of each sample as well as its refractive index in the $300 \mathrm{~nm}-2 \mu \mathrm{m}$ spectral range using a J.A. Woollam VASE spectroscopic ellipsometer and accompanied WVASE fitting software. The gold film thicknesses of those samples are measured to be 44,21 , and $11 \mathrm{~nm}$. The measured values for the real $(n)$ and imaginary $(k)$ parts of the refractive index for each of the three samples can be found in Supplemental Material [42], see data files. The relative permittivity values $\varepsilon_{2}(\lambda)=(n-j k)^{2}$ of the thin gold films used in all our calculations are extrapolated from these files. In calculating $\varepsilon_{2}(\lambda)$ for gold films of thickness $d$ other than 44,21 , or $11 \mathrm{~nm}$, a linear interpolation is used $(d$ is in nanometers): 


$$
\varepsilon_{2, d}= \begin{cases}\varepsilon_{2,11 \mathrm{~nm}}, & d \leq 11, \\ \varepsilon_{2,11 \mathrm{~nm}}+\left(\varepsilon_{2,21 \mathrm{~nm}}-\varepsilon_{2,11 \mathrm{~nm}}\right) \frac{d-11}{21-11}, & 11<d \leq 21, \\ \varepsilon_{2,21 \mathrm{~nm}}+\left(\varepsilon_{2,44 \mathrm{~nm}}-\varepsilon_{2,21 \mathrm{~nm}}\right) \frac{d-21}{44-21}, & 21<d \leq 44, \\ \varepsilon_{2,44 \mathrm{~nm}}, & 44<d,\end{cases}
$$

as in the case of solving Eqs. (B4) and (B5) for the longrange SPP and APP root locus or calculating Eq. (B3) to retrieve $r$ for Figs. 4 and 7 and Movie 4.

The relative permittivity of the glass coupling prisms $\varepsilon_{1}$ is calculated from a Sellmeier equation for uncoated fused silica provided by the prism manufacturer (ThorLabs):

$$
\varepsilon_{1}(\lambda)=1+\sum_{m=1}^{3} \frac{b_{m} \lambda^{2}}{\lambda^{2}-c_{m}}
$$

where $b_{1}=0.6961663, b_{2}=0.4079426, b_{3}=0.8974794$, $c_{1}=0.068404^{2}, c_{2}=0.1162414^{2}, c_{3}=9.896161^{2}$, and $\lambda$ is the wavelength in microns. The relative permittivity of air $\varepsilon_{3}$ and the relative permeabilities of all media $\mu_{i}$ $(i=1,2,3)$ are taken to be 1 .

\section{Calculating the long-range SPP and APP dispersion relations}

The field amplitude reflection coefficient of a thin gold film can be derived by summing reflected wave contributions from the front interface with those of internal reflections from within the film $[3,9]$ :

$$
\begin{aligned}
r\left(k_{z}, \omega\right) & =r_{12}+\frac{t_{12} t_{21}}{r_{21}} \sum_{m=1}^{\infty}\left(r_{21} r_{23} e^{-2 j k_{x_{2}} d}\right)^{m} \\
& =\frac{r_{12}+r_{23} e^{-2 j k_{x_{2}} d}}{1-r_{21} r_{23} e^{-2 j k_{x_{2}} d}},
\end{aligned}
$$

where $\quad r_{i j}=\left(k_{x, i} / \varepsilon_{i}-k_{x, j} / \varepsilon_{j}\right) /\left(k_{x, i} / \varepsilon_{i}+k_{x, j} / \varepsilon_{j}\right) \quad$ and $t_{i j}=1+r_{i j}$ are Fresnel reflection and transmission coefficients from medium $i$ to medium $j, \varepsilon_{i}$ and $\mu_{i}$ are the relative permittivity and permeability of the glass substrate $(i=1)$, gold film $(i=2)$, and free space $(i=3)$, respectively, $d$ is the gold film thickness, $\omega$ is the angular frequency, $c$ is the speed of light in vacuum, and $k_{z}$ and $k_{x}$ are the parallel $(z)$ and normal $(x)$ wave-vector components to the front interface, respectively, satisfying the relation $k_{x, i}^{2}+k_{z}^{2}=(\omega / c)^{2} \varepsilon_{i} \mu_{i}$ imposed by the wave equation in each medium.

The electromagnetic modes are the field solutions for which all internal reflections constructively interfere such that $r$ diverges (resonant field solutions):

$$
1-r_{21} r_{23} e^{-2 j k_{x_{2}} d}=0,
$$

whereas the antiresonant field solutions satisfy that all internal reflections destructively interfere with the initial reflection from the front interface such that $r$ vanishes:

$$
r_{12}+r_{23} e^{-2 j k_{x_{2}} d}=0 .
$$

The long-range SPP and APP dispersion curves and root loci, plotted in Figs. 3, 4, and 7 and Movie 4, are calculated by numerically solving Eqs. (B4) and (B5) over the appropriate wave-vector and spectral $(300 \mathrm{~nm}-2 \mu \mathrm{m})$ ranges. The method to determine the appropriate wavevector range for a given angular range is detailed in Appendix B 6. The film thickness and permittivity values used in calculations are experimentally measured as detailed in Appendix B 3.

\section{Calculating the predicted reflectance signature}

To calculate the predicted reflectance from our samples, we follow the primary beam path inside the coupling prism, as depicted in Fig. 6. The beam collected by the detection arm is obtained by a sequence of three scattering interactions: a transmission into the prism through its first front facet, a subsequent reflection from the prism back facet on which the thin gold film is fabricated, and a final transmission out of the prism through the second prism front facet.

Consequently, the intensity of the beam along its path is reduced compared to that of the impinging beam at three separate instances. Initially, the beam propagates in free

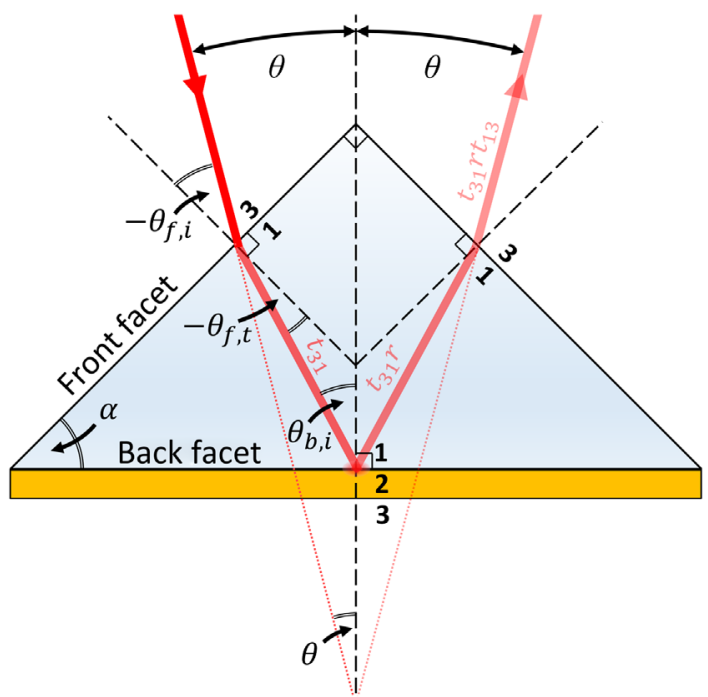

FIG. 6. Path traveled by light in the coupling prism. The reflected beam (red) undergoes the primary beam path in the glass prism, consisting of transmission through the first front facet $\left(t_{31}\right)$, reflection from the back facet $(r)$, and transmission through the second front facet $\left(t_{13}\right)$. The incidence $\left(\theta_{i}\right)$ and transmission $\left(\theta_{t}\right)$ angles in each passage are taken as positive if the beam propagation direction is counterclockwise to the facet normal (dashed black lines) and negative otherwise. 

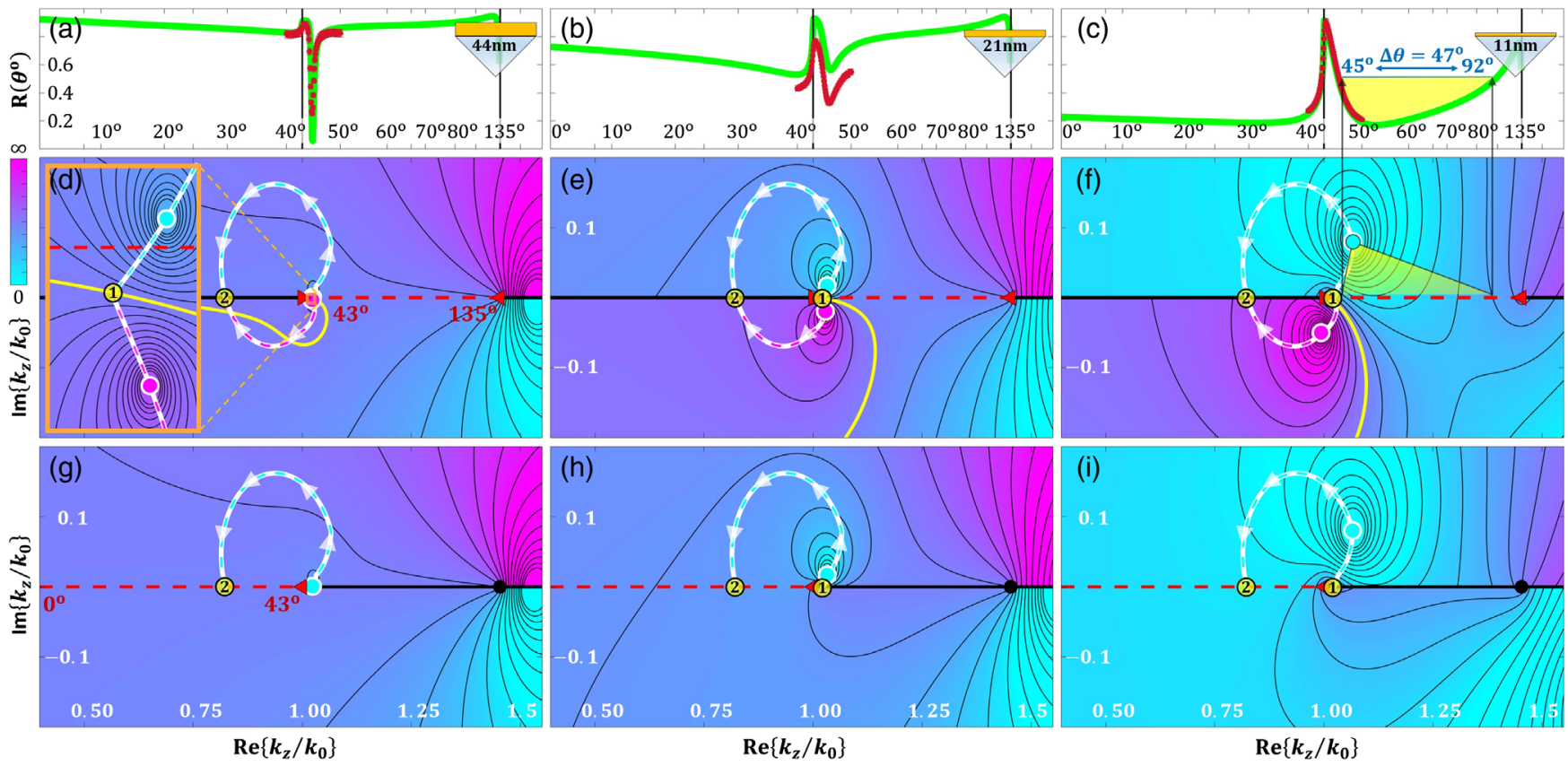

FIG. 7. Closed-loop root locus of colocalized SPP-APP pairs. (a)-(c) Measured (red) and predicted (green) reflectance of a gold film illuminated through a glass coupling prism as a function of incidence angle $\theta$ at illumination wavelength $\lambda=800 \mathrm{~nm}$ for film thickness (a) 44, (b) 21, and (c) $11 \mathrm{~nm}$. (d)-(j) Field amplitude reflection coefficient magnitude $|r|$ of the respective gold films (log scale) as a function of the complex longitudinal wave vector $k_{z}$, calculated in the topological neighborhood of the two observable real-axis segments (red dashed lines): (d)-(f) above and (h)-(j) below the critical angle. White curves denote the calculated root locus of the reflection pole (magenta) and zero (cyan) points, corresponding to the long-range SPP and APP, as the film thickness decreases from $\infty \rightarrow 0$ (points $1 \rightarrow 2$ ). Vertical black lines in (a)-(c) and black dots in (d)-(j) denote the critical $\left[\theta \approx 43^{\circ}, k_{z}=k_{0}=(\omega / c)\left(\varepsilon_{3} \mu_{3}\right)^{1 / 2}\right]$ and grazing $\left[\theta \approx 135^{\circ}, k_{z}=(\omega / c)\left(\varepsilon_{1} \mu_{1}\right)^{1 / 2}\right]$ angles. The wideband absorption dip formation is illustrated by yellow regions in (c) and (f). The orange rectangle in (d) marks an enlarged section. The $|r|=1$ contour line in (d)-(j) is colored yellow. Movie 4 shows the continuous transition from points $1 \rightarrow 2$.

space at a predefined angle $\theta$ relative to the prism back facet normal (vertical black dashed line). It then impinges on the prism front facet at an angle $\theta_{f, i}=\theta-\alpha$ relative to that front facet normal (inclined black dashed line), where $\alpha=$ $45^{\circ}$ is the prism base angle, and is partially transmitted into the prism at an angle $\theta_{f, t}$ determined by Snell's law $\left(\varepsilon_{3} \mu_{3}\right)^{1 / 2} \sin \left(\theta_{f, i}\right)=\left(\varepsilon_{1} \mu_{1}\right)^{1 / 2} \sin \left(\theta_{f, t}\right)$. Hence, its amplitude is reduced by a factor of the Fresnel transmission coefficient $t_{31}\left(k_{z^{\prime}}, \omega\right)=1+r_{31}\left(k_{z^{\prime}}, \omega\right)$ calculated for the wave-vector component parallel to the prism front facet $k_{z^{\prime}}=(\omega / c)\left(\varepsilon_{3} \mu_{3}\right)^{1 / 2} \sin \left(\theta_{f, i}\right)$. Subsequently, the beam propagates within the prism until it impinges on the thin gold film at an incidence angle $\theta_{b, i}=\theta_{f, t}+\alpha$ relative to the prism back facet normal. Its amplitude is therefore again reduced by the thin gold film reflection coefficient $r\left(k_{z}, \omega\right)$ calculated using Eq. (B3) for the wave-vector component parallel to the back facet $k_{z}=(\omega / c)\left(\varepsilon_{1} \mu_{1}\right)^{1 / 2} \sin \left(\theta_{b, i}\right)$. Because the prism is symmetric, the beam escapes the prism at an opposite angle $\theta$ to which it enters, and its amplitude is yet again reduced by $t_{13}\left(k_{f}, \omega\right)$. Overall, the reflected beam collected by the detector is therefore reduced in intensity relative to the incident beam by a factor of the reflectance:

$$
R(\theta, \lambda)=\left|t_{31}\left(k_{z^{\prime}}, \omega\right) r\left(k_{z}, \omega\right) t_{13}\left(k_{z \prime}, \omega\right)\right|^{2} .
$$

The predicted reflectance signatures, plotted in Figs. 3, 4, and 7 and Movie 4, are all derived using Eq. (B6).

\section{SPP-APP pair trajectory in momentum-energy space}

The reflectance at each incidence angle $\theta$ is determined by the magnitude of the thin film field amplitude reflection coefficient $r\left(k_{z}, \omega\right)$ at a corresponding point $k_{z}$ that satisfies (see Appendix B 5)

$$
\begin{aligned}
k_{z} & =(\omega / c) \sqrt{\varepsilon_{1} \mu_{1}} \sin \left(\theta_{b, i}\right), \\
\sin (\theta-\alpha) & =\sqrt{\varepsilon_{1} \mu_{1} / \varepsilon_{3} \mu_{3}} \sin \left(\theta_{b, i}-\alpha\right),
\end{aligned}
$$

following Eqs. (B3) and (B6). Assuming a lossless glass prism, the values of $r\left(k_{z}, \omega\right)$ over the real $k_{z}$ axis are therefore sufficient to predict the reflectance. Our approach is to identify how $r\left(k_{z}, \omega\right)$ over the real $k_{z}$ axis is affected by the distribution of its discrete modes and discrete antiresonant field solutions, associated with the poles and zeros over the complex $k_{z}$ domain, respectively. The closer a pole or zero is to the real $k_{z}$ point associated with a 
given incidence angle, the stronger its signature over the observed reflectance at that angle.

This measure of closeness is not obvious due to the nontrivial topology of the complex wave-vector domain. The quadratic relation between parallel and normal wavevector components $k_{x, i}^{2}+k_{z}^{2}=(\omega / c)^{2} \varepsilon_{i} \mu_{i}$ implies that $r\left(k_{z}, \omega\right)$ is a multivalued function of $k_{z}$, so Eq. (B3) yields four different values for the multiple root branches of $k_{x, 1}$ and $k_{x, 3}$. The standard mathematical procedure for retrieving a single-valued function for $r\left(k_{z}, \omega\right)$ is to extend the $k_{z}$ domain over four separate Riemann sheets, associated with the proper $\left(\operatorname{Im} k_{x} \leq 0\right)$ and improper $\left(\operatorname{Im} k_{x} \geq 0\right)$ root branches for each of $k_{x, 1}$ and $k_{x, 3}$. These sheets are topologically connected at the two branch cuts $\left(\operatorname{Im} k_{x, 1}=0\right.$ and $\left.\operatorname{Im} k_{x, 3}=0\right)$ across which $r\left(k_{z}, \omega\right)$ is continuous [9]. Hence, $r\left(k_{z}, \omega\right)$ becomes a continuous single-valued function over a topologically nontrivial wave-vector domain.

The real $k_{z}$ point associated with any given incidence angle must lie in the $k_{x, 1}$-proper $k_{x, 3}$-proper sheet to describe physical waves that carry energy along their direction of propagation in the substrate and superstrate. However, the spatial decomposition of the total field [Eqs. (A1) plus (A2)] requires only that the $-\infty$ to $\infty$ integration end points be in the proper-proper sheet to recover a physical field. The integration path itself can be continuously deformed in the complex $k_{z}$ domain across all sheets with the field remaining unchanged [9]. Hence, while the Fourier spectrum (real $k_{z}$ ) is unique, the Laplace spectrum is not (complex $k_{z}$ ), and so complex poles and zeros across all sheets can contribute to the observed field. To evaluate the contribution of each pole or zero, we introduce a notion of topological proximity to the directly observable part of the wave-vector domain, as illustrated in Fig. 7.

There are two distinct segments of the real $k_{z}$ axis that are directly accessible from the far field using the coupling prism (red dashed lines). The first segment shown in Figs. 7(h)-7(j) runs along the branch cuts of both $k_{x, 3}$ and $k_{x, 1}$, up to the branch point of $k_{x, 3}$ at the free-space light line $k_{z}=(\omega / c)\left(\varepsilon_{3} \mu_{3}\right)^{1 / 2}$. It corresponds to incidence angles ranging from normal incidence onto the prism $\left(\theta=0^{\circ}\right)$ to the critical angle for total internal reflection at the prism back facet $\left(\theta \approx 43^{\circ}\right)$. The second segment shown in Figs. 7(d)-7(f) runs only along the branch cut of $k_{x, 1}$ between the branch points of $k_{x, 3}$ and $k_{x, 1}$, ending at the glass substrate light line $k_{z}=(\omega / c)\left(\varepsilon_{1} \mu_{1}\right)^{1 / 2}$. It corresponds to incidence angles ranging from the critical angle $\left(\theta \approx 43^{\circ}\right)$ to the grazing angle at the prism back facet $\left(\theta_{b, i}=90^{\circ}, \theta \approx 135^{\circ}\right)$, in between the two vertical black lines in Figs. 7(a)-7(c).

Each of the two directly observable wave-vector segments has a different topological neighborhood in the complex $k_{z}$ domain for which $r\left(k_{z}, \omega\right)$ remains continuous across branch cuts. While toward the upper half space $\left(\operatorname{Im} k_{z}>0\right)$ the topological neighborhood of both segment consists of the proper-proper sheet $\left(\operatorname{Im} k_{x, 1} \leq 0, \operatorname{Im} k_{x, 3} \leq 0\right)$, to continuously reach the lower half space $\left(\operatorname{Im} k_{z}<0\right)$ through each segment requires crossing different branch cuts and, therefore, arrives at different sheets. The first segment crosses both branch cuts into the improperimproper sheet $\left(\operatorname{Im} k_{x, 1} \geq 0, \operatorname{Im} k_{x, 3} \geq 0\right)$ [Figs. 7(h)-7(j)], whereas the second segment crosses only the $k_{x, 1}$ branch into the improper-proper sheet $\left(\operatorname{Im} k_{x, 1} \geq 0, \operatorname{Im} k_{x, 3} \leq 0\right)$ [Figs. 7(d)-7(f)]. Consequently, the two observable segments probe different poles and zeros, as each segment is affected only by those poles and zeros topologically adjacent to it.

To map the contribution of the long-range SPP and APP to the reflectance, we track the path of their associated pole and zero points in the topologically nontrivial complex $k_{z}$ domain [Figs. 7(d)-7(j)]. The root loci (white curves) of their respective pole (magenta) and zero (cyan) points jointly form a semiclosed loop: They originate in common creation or annihilation at the thick film limit (yellow point 1) and end in coalescence over the Brewster angle of the emerging glass-air interface at the vanishing film limit (yellow point 2). At point 1, both the pole and zero lie in the improper-proper sheet and so cancel as they meet, signifying that both resonant and antiresonant plasmonic degrees of freedom are eliminated once the gold-air interface on which they are localized becomes inaccessible. Conversely, at point 2 , the zero has already crossed the real axis into the proper-proper sheet, so the pole and zero occur at the same $k_{z}$ but on opposite sheets. Consequently, reciprocity dictates that their associated field solutions become identical, and the two degrees of freedom localized on the vanishing gold-air interface coalesce into the single antiresonant degree of freedom available for the emerging glass-air interface at the Brewster angle.

The contribution of the long-range SPP and APP to the reflectance depends on which stage the pole and zero are located along their closed-loop root locus. There are three stages connecting four critical points: the creation point, the point at which the zero crosses between sheets along the real $k_{z}$ axis, the point of maximum momentum splitting between the zero and pole, and the coalescence point. In the first stage, the zero moves in close topological proximity to the real axis segment observable above the critical angle [44 nm, Fig. 7(d)], and the narrow Kretschmann reflectance dip emerges as a result [Fig. 7(a)]. In the second stage, the zero and pole lie on opposite sheets at comparable topological proximity to that same observable segment $[21 \mathrm{~nm}$, Fig. 7(e)], so the dip-dominated reflectance gives way to a combined peak-and-dip signature [Fig. 7(b)]. In the third stage, the zero and pole move away from the topological neighborhood of that segment, retrieving the flat total internal reflection response of a bare glass prism above the critical angle, while the zero also moves into close topological proximity of the other observable real axis segment retrieving the standard Brewster angle signature below the critical angle. 
The strong wideband absorption we observe for ultrathin gold films [Fig. 3(c)] originates in the rapid approach of the zero and pole to the point of maximal momentum splitting [11 nm, Fig. 7(f)], prior to their exponential convergence toward the coalescence point in the vanishing film thickness limit. In the process, the reflectance peak squeezes onto the critical angle, because (i) the influence of the pole shifts to lower incidence angles as it travels to smaller wave vectors under the free-space light line, while (ii) its influence is also cut off at the critical angle, as it travels in the improper-proper sheet disconnected from the topological neighborhood of the real axis segment observable below the critical angle. Conversely, the influence of the zero extends over the entire angular range, because it travels in the proper-proper sheet. Consequently, the reflectance dip extends over most of the angular range above the critical angle [Fig. 7(f), highlighted in yellow], giving rise to strong angularly and spectrally wideband absorption: an approximately $83 \%$ absorption dip with a FWHM angular bandwidth of $\Delta \theta \approx 47^{\circ}$ [Fig. 7(c), highlighted in yellow, shown for $\lambda=800 \mathrm{~nm}]$ and spectral bandwidth of $\Delta \lambda>$ $1.34 \mu \mathrm{m}$ [Fig. 3(c), from $\lambda \approx 655 \mathrm{~nm}$ and extending beyond our measurement range]. Appendix B 9 details how to design for this effect.

\section{The excitation direction dependence of APPs}

In principle, each plasmonic interface in a structure contributes a pair of resonant (SPPs) and antiresonant (APPs) degrees of freedom that are jointly created and annihilated in momentum-energy space, excluding the interface facing the source. For example, the thin gold film has two plasmonic interfaces, supporting one resonantantiresonant pair that originates from its back interface relative to the direction of illumination. As shown in Fig. 8, when illuminated from the glass side, one APP exists, and it is paired to the long-range SPP localized on the metal-air interface. However, when illuminated from the air side, one different APP exists, and it is paired to the short-range SPP localized on the gold-glass interface. These two separate APPs can be termed "long-range" and "short-range" by association, and, while the former can be observed in the far field using a coupling prism (Fig. 3), the latter typically requires excitation with larger momentum. This example illustrates that, unlike resonant DOFs, antiresonant DOFs are directional and depend on the direction from which the structure is illuminated.

\section{Mutual deflection in the line shape of an SPP-APP pair}

An isolated complex reflection pole or zero projects an extremum onto the angular line shape at the angle corresponding to the real part of its wave vector. In contrast, when both a complex pole (SPP) and zero (APP) are present, the extrema they project become offset from the real part of their wave vectors by an amount that grows with

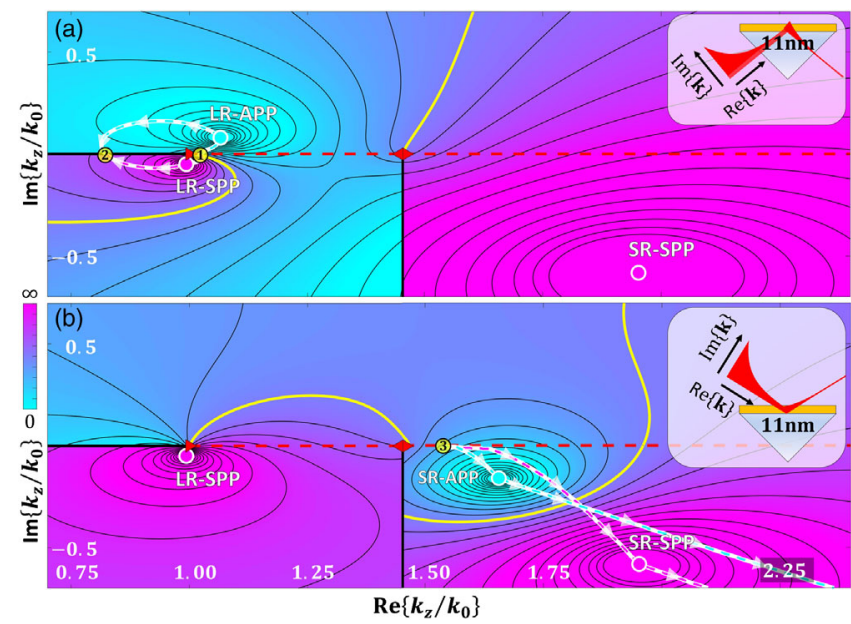

FIG. 8. Dependence of APPs on the direction of illumination. The field amplitude reflection coefficient $r$ of an $11 \mathrm{mn}$ gold film at illumination wavelength $\lambda=800 \mathrm{~nm}$ calculated over the complex wave-vector range $k_{z}$ observable when illuminating from (a) the glass prism side and (b) the air side. SPPs and APPs correspond to exact reflection pole (magenta) and zero (cyan) wave vectors (white circles), respectively. Unlike SPPs, the supported APPs depend on the illumination direction: In (a), a "long-range" APP is paired to the long-range SPP, and in (b), a different "short-range" APP is paired to the short-range SPP (white curves denote their calculated root locus as the film thickness decreases from $\infty \rightarrow 0$ ). The reflection coefficient is calculated in the topological neighborhood of the real wavevector axis for illumination with wave vectors exceeding the light line in air (left dashed red line) and glass (right dashed red line), with branch cuts denoted by black lines. The $|r|=1$ contour line is colored yellow.

the imaginary part. For instance, taking a simple rational function approximation to the reflection coefficient Eq. (C2) yields that the maximum (+) and minimum $(-)$ in the reflectance Eq. (C3)

$\Omega_{ \pm}=-\frac{Q}{2}\left(1 \pm \sqrt{1+\left(\frac{2}{Q}\right)^{2}}\right) \stackrel{Q \ll 2}{\approx} Q^{-1}, \quad-Q-Q^{-1}$

are offset from the real part of their normalized wave vectors by an amount that scales with the imaginary part $\left( \pm Q^{-1}\right)$, where $Q=q+(b-1) / q$ using the quantities defined in Eq. (C4). This mutual deflection is evident from tracing contour lines in Figs. 4(h) and 4(f) for the $11 \mathrm{~nm}$ gold film, at which the long-range SPP and APP wave vectors attain much larger imaginary parts compared to the 21 and $44 \mathrm{~nm}$ films. It also explains the offset in Fig. 3(f) between the measured high and low reflectance areas and the calculated real part of the dispersion. In principle, therefore, to understand a forced optical response in terms of discrete SPP-APP pairs, one needs to consider their fullfledged complex dispersion. 


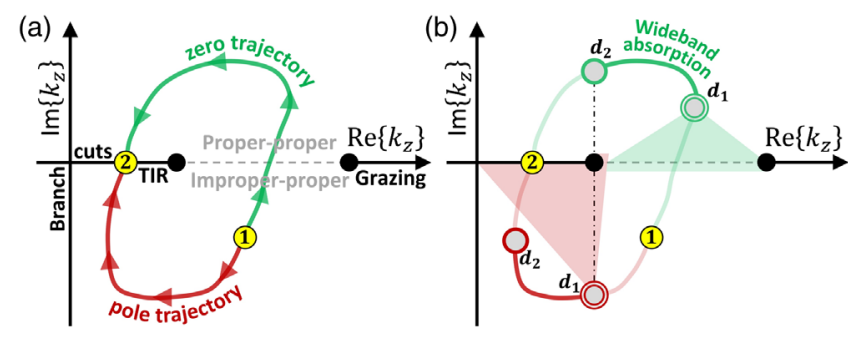

FIG. 9. SPP-APP pair location range for wideband absorption. (a) Sketch of the long-range SPP-APP pair (red-green) closedloop root locus in the complex wave-vector domain $\left(k_{z}\right)$ as the film thickness decreases $(\infty \rightarrow 0)$, from creation or annihilation at the gold-air single-interface SPP wave vector to coalesce at the glass-air Brewster angle $(1 \rightarrow 2)$. (b) The tilt of their associated pole-zero pair (circles) with respect to the real axis ensures a film thickness range $d_{1} \rightarrow d_{2}$ for which the pole crossed under the total-internal-reflection (TIR) branch point and is mostly blocked from the observable (proper-proper) real (angular) axis, but the zero has not yet crossed above it, thus imprinting an absorption dip ranging from the critical angle to the grazing angle.

\section{Designing for the black gold film effect}

The phenomenon of wideband absorption by ultrathin gold films can be predicted from the shape of the longrange SPP-APP pair root locus as the film thickness decreases (Fig. 9). This shape is dictated by the root locus end points, at the asymptotic film thickness limits when the gold-air interface on which the pair is localized becomes inaccessible to the source. As illustrated in Fig. 9(a), the root locus starts with generation of the reflection polezero pair at the gold-air single-interface SPP wave vector $k_{z, d \rightarrow \infty}=k_{0}\left[\varepsilon_{2} \varepsilon_{3} /\left(\varepsilon_{2}+\varepsilon_{3}\right)\right]^{1 / 2}$ in the improperproper sheet and ends with a coalesce at the glass-air Brewster angle $k_{z, d \rightarrow 0}=k_{0}\left[\varepsilon_{1} \varepsilon_{3} /\left(\varepsilon_{1}+\varepsilon_{3}\right)\right]^{1 / 2}$ to which the pole and zero converge from opposite sheets (see Appendix B 6).

At any stage along the root locus, the pole-zero pair must be tilted with respect to the real wave-vector axis $\left(\operatorname{Re}\left\{k_{z, L R-A P P}\right\}>\operatorname{Re}\left\{k_{z, L R-S P P}\right\}\right)$ to satisfy a smaller than one reflectance (see Supplemental Material [42]). Hence, as illustrated in Fig. 9(b), there exists a range of thicknesses $d_{1} \leq d<d_{2}$ for which the zero crosses under the branch point of total internal reflection in the prism $\left(\operatorname{Re}\left\{k_{z, \text { LR-SPP }}\right\} \leq k_{0} n_{3}\right)$, but the zero does not yet cross above it $\left(\operatorname{Re}\left\{k_{z, \mathrm{LR}-\mathrm{APP}}\right\}>k_{0} n_{3}\right)$. In that range, the pole is blocked from strongly projecting onto the observable (proper-proper) angular range anywhere away from the critical angle, while the zero remains to dominate the entire range from the critical angle to grazing angle in the prism. Solely based on that, one can predict a wide absorption dip for a narrow range of film thicknesses in the ultrathin regime.

The film thickness achieving the strongest wideband absorption within that range satisfies the condition that the pole is located under the total internal reflection branch point [film thickness $d_{1}$ in Fig. 9(b)]:

$$
\operatorname{Re}\left\{k_{z, \mathrm{LR}-\mathrm{SPP}}\right\}=k_{0} n_{3},
$$

where $n_{3}$ is the refractive index of the superstrate $\left(n_{3}=1\right.$ for air). This condition maximizes $\operatorname{Re}\left\{k_{z, \mathrm{LR}-\mathrm{APP}}\right\}$ in the range $d_{1} \leq d<d_{2}$ [Fig. 9(b)] and with it the projection strength of the zero over the angular range above the critical angle-maximizing absorption (a maximally split state). One thus needs to solve Eq. (B4) under the constraint Eq. (B9), finding the root of a scalar real-valued function of one real variable

$\left.f(a)\right|_{a \in \mathbb{R}^{+}}=\left.\operatorname{Re}\left\{\ln \left(r_{21} r_{23}\right) / k_{x, 2}\right\}\right|_{k_{z}=k_{0}\left(n_{3}-j a\right)}=0$,

and substitute the solution in the closed-form expression

$$
d_{1}=\ln \left(r_{21} r_{23}\right) /\left.2 j k_{x, 2}\right|_{k_{z}=k_{0}\left(n_{3}-j a\right)},
$$

which yields a real value, since $k_{z}$ now solves Eq. (B4).

We design the ultrathin gold film that exhibits strong wideband absorption [Figs. 3(c) and 7(c)] using Eqs. (B10) and (B11). Subject to fabrication process variation, we aim for a film thickness at which the long-range SPP-APP pair satisfies Eq. (B9) at $\lambda=800 \mathrm{~nm}$, so the resulting polezero positions in Figs. 7(f) and 7(h) mimic condition $d_{1}$ in Fig. 9(b). Despite fixing the wavelength, this procedure also yields spectrally wideband absorption, because gold dispersion decreases monotonically at larger wavelengths away from the interband transitions at $520 \mathrm{~nm}$. Consequently, it takes a wide spectral range for the SPPAPP locations to move across the entire range of positions suitable for wideband absorption $\left(d_{1} \geq d<d_{2}\right)$. As seen in Fig. 3(f), the fabricated sample satisfies condition $d_{1}$ at $\lambda=1718 \mathrm{~nm}$ when $\operatorname{Re}\left\{k_{z, \mathrm{LR} \text {-SPP }}\right\}$ (solid black curve) crosses the light line $k_{0} n_{3}$ (dashed curve) and satisfies condition $d_{2}$ at $\lambda=546 \mathrm{~nm}$ when $\operatorname{Re}\left\{k_{z, \mathrm{LR}-\mathrm{APP}}\right\}$ (white curve) crosses the light line-a bandwidth of about $\Delta \lambda=$ $1.17 \mu \mathrm{m}$ (FWHM estimation is larger).

One can further increase the spectral bandwidth by maximizing the path $d_{1} \rightarrow d_{2}$, that is, finding the film thickness at which $\operatorname{Re}\left\{k_{z, \mathrm{LR}-\mathrm{APP}}\right\}$ under condition $d_{1}$ is maximized over a range of wavelengths

$$
\max _{\lambda \text { in range }}\left(\left.\operatorname{Re}\left\{k_{z, \text { LR-APP }}\right\}\right|_{d=d_{1}(\lambda)}\right),
$$

adding an optimization layer on top of Eq. (B10) that maximizes a real function over a bounded domain. In each iteration, Eq. (B10) is solved at a given wavelength for $k_{z, \mathrm{LR}-\mathrm{SPP}}$ that satisfies condition $d_{1}$, followed by extracting $k_{z, \text { LR-APP }}$ by solving Eq. (B5) using $k_{z, \text { LR-SPP }}^{*}$ as an initial guess for fast convergence. The entire calculation can be completed with the computational complexity of $\mathrm{O}\left[\log \left(\text { error }^{-1}\right)^{2}\right]$, as the root finding Eq. (B10) can be implemented with at least a quadratic convergence rate and 
the maximization Eq. (B12) with at least a linear convergence rate. Moreover, it may suffice to solve Eq. (B10) once at a single wavelength, since performing the maximization over a spectral range changes our planned film thickness by only about $1 \mathrm{~nm}$ (comparable to fabrication error), improving the expected wavelength-averaged absorption cross section by less than $1 \%$.

\section{APPENDIX C: INABILITY OF THE FANO MODEL TO DESCRIBE ULTRATHIN FILM REFLECTANCE}

The notion of a Fano resonance is often invoked to explain asymmetrical peak-and-dip line shapes in the spectrum of driven optical systems. It is defined by the following spectral line shape formula $[10,11]$ :

$$
\sigma(E)=D^{2} \frac{(q+\Omega)^{2}}{1+\Omega^{2}},
$$

where $E$ is the energy, $q$ is the Fano parameter, $\Omega=$ $2\left(E-E_{0}\right) / \Gamma$ is the dimensionless frequency, $\Gamma$ and $E_{0}$ are the resonance width and energy, respectively, and $D=$ $2 \sin \delta$ is related to the Fano parameter by $q=\cot \delta$. The Fano resonance emerges in the response of two coupled oscillators (states) to a driving force acting on one of them. However, such a response conforms to the Fano line shape only if the two oscillators are weakly coupled and have vastly different damping rates, corresponding to a discrete state and a continuum [10].

While the Fano model typically relates to spectral line shapes, it can also be fitted to angular line shapes, meaning to scattering responses that depend on momentum rather than energy. Such a Fano model has been invoked to explain the asymmetric angular line shape occurring in the reflectance of thin metal films in attenuated total reflection conditions, specifically those associated with the zeroreflectance Kretschmann dip [50,51]. It associates the asymmetric Kretschmann dip signature with a Fano resonance emerging from the coupling between the long-range SPP mode (a narrow discrete state) and the continuum of scattered background radiation. However, as shown below, the Fano model cannot predict nor explain the wideband absorption we observe for ultrathin gold films.

\section{Approximating the angular reflectance with a Fano model}

To argue that the asymmetric reflectance signature of thin metal films arises from a Fano resonance, the field amplitude reflection coefficient $r$ is approximated in the angular range of observation as a function of momentum to include only the straightforward contribution of a nearby zero and pole [50]:

$$
r\left(k_{z}\right) \approx D \frac{k_{z}-k_{z, \text { zero }}}{k_{z}-k_{z, \text { pole }}},
$$

where $k_{z}$ is the complex parallel wave-vector component defined in Appendix B 5 and $k_{z \text {,pole }}$ and $k_{z, \text { zero }}$ are complex parameters that should fit the dominant reflection pole and zero locations in the wave-vector domain, respectively. This approximation produces the angular reflectance line shape given by the squared absolute value of Eq. (C2), which obeys a generalized Fano equation

$$
\begin{aligned}
R(\theta) & \approx D^{2} \frac{\left(k_{z}-\operatorname{Re} k_{z, \text { zero }}\right)^{2}+\left(\operatorname{Im} k_{z, \text { zero }}\right)^{2}}{\left(k_{z}-\operatorname{Re} k_{z, \text { pole }}\right)^{2}+\left(\operatorname{Im} k_{z, \text { pole }}\right)^{2}} \\
& =D^{2} \frac{(q+\Omega)^{2}}{1+\Omega^{2}}+D^{2} \frac{b}{1+\Omega^{2}}
\end{aligned}
$$

in which the dimensionless frequency $\Omega$ and Fano parameter $q$ are joined by a screening parameter $b$ :

$$
\begin{aligned}
\Omega & \triangleq\left(k_{z}-\operatorname{Re} k_{z, \text { pole }}\right) / \operatorname{Im} k_{z, \text { pole }} \\
q & \triangleq \operatorname{Re}\left\{k_{z, \text { pole }}-k_{z, \text { zero }}\right\} / \operatorname{Im} k_{z, \text { pole }} \\
b & \triangleq\left(\operatorname{Im} k_{z, \text { zero }} / \operatorname{Im} k_{z, \text { pole }}\right)^{2}
\end{aligned}
$$

and $k_{z}$ is real to correspond to incidence angles $\theta$ according to Eq. (B7) in Appendix B 6.

Before discussing the invalidity of this approximation for ultrathin films, we emphasize that the generalized line shape Eq. (C3) differs from the exact Fano line shape given by Eq. (C1): In addition to the Fano contribution, it includes a non-negative Lorentzian term that depends on the screening parameter. While not affecting the resonance width, the screening parameter does determine its contrast and prevents it from reaching zero [55]. Hence, the generalized line shape Eq. (C3) is dominated by the Fano resonance term only if the imaginary part of the pole is significantly larger than that of the zero (high contrast):

$$
\left|\operatorname{Im} k_{z, \text { zero }}\right| \ll\left|\operatorname{Im} k_{z, \text { pole }}\right| .
$$

Moreover, within a finite angular range $\left( \pm\left|\operatorname{Im} k_{z, \text { zero }}\right|\right)$ of the Fano resonance $\operatorname{dip}\left(k_{z}=\operatorname{Re} k_{z, \text { zero }}\right)$, the dominant contribution to the line shape is necessarily from the peak of the Lorentzian term

$$
\left|k_{z}-\operatorname{Re} k_{z, \text { zero }}\right|<\left|\operatorname{Im} k_{z, \text { zero }}\right|,
$$

and that range broadens as the contrast deteriorates.

The asymmetric zero-reflectance Kretschmann dip forms (roughly $50 \mathrm{~nm}$ in gold) at the film thickness for which the zero crosses the real axis $\left[\operatorname{Im} k_{z, \text { zero }}=0\right.$, Figs. 7(d) and 7(h)], coinciding with the condition under which the approximate reflectance Eq. (C3) reduces to an exact Fano line shape Eq. $(\mathrm{C} 1)(b=0)$. The high contrast emerging in that case allows the Fano resonance term to be well fitted to the pronounced reflectance dip. However, for thinner films (below $25 \mathrm{~nm}$ in gold), the strong coupling between the 

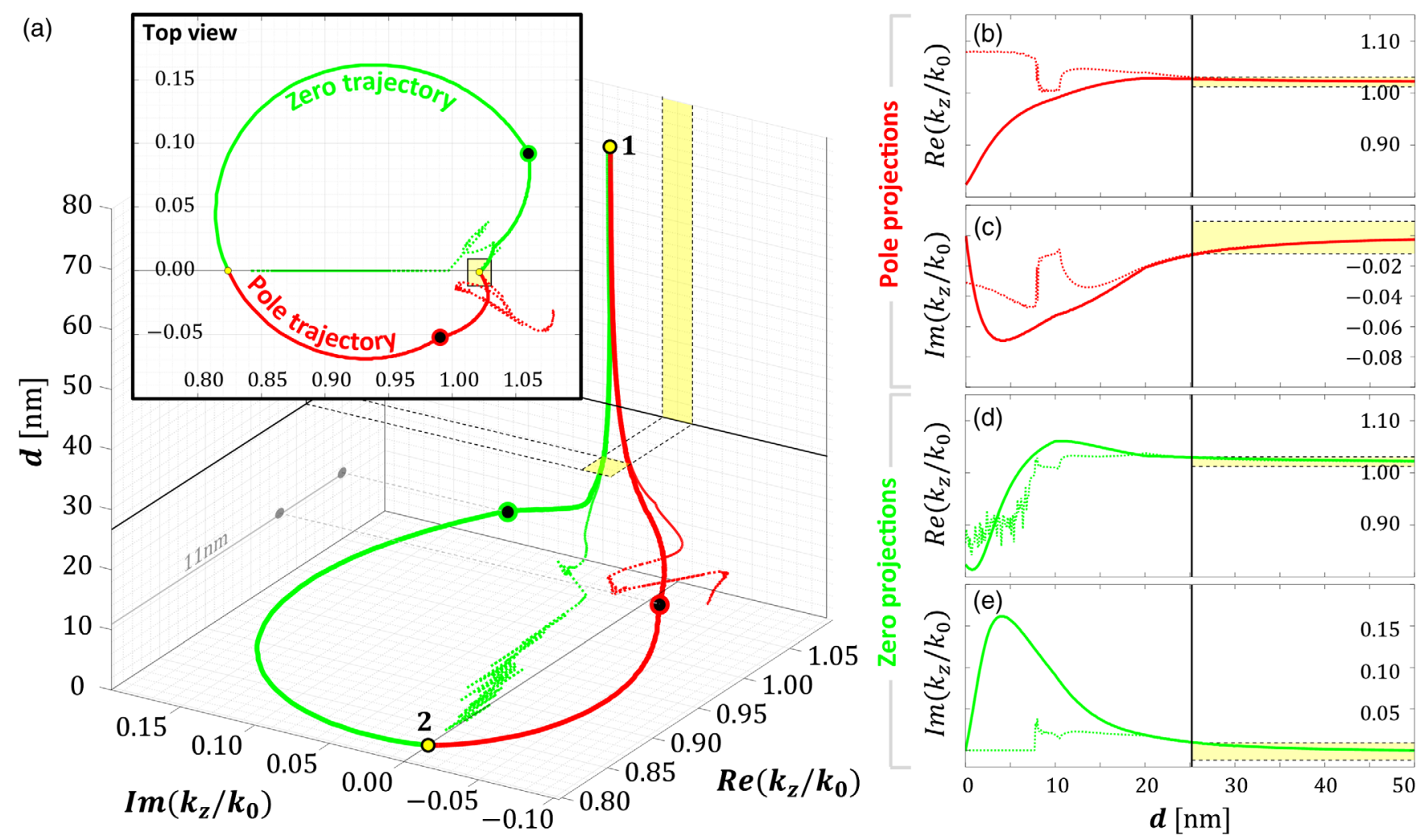

FIG. 10. Comparison of the exact and Fano fitted dispersion. (a) The exact (solid curves) and fitted (dotted curves) values of the reflection pole (red) and zero (green) complex wave vectors as a function of gold film thickness $d$ at illumination wavelength $\lambda=800 \mathrm{~nm}$. Inset: A top view showing their closed-loop trajectory (yellow points $1 \rightarrow 2$ ). (b)-(e) Projections of the pole and zero wave vectors in (a) over the real and imaginary axes separately. The narrow wave-vector region in which the generalized Fano line shape fits the exact calculated reflectance with a reasonable coefficient of determination $\left(R^{2}>0.7\right)$ is highlighted in yellow, corresponding to films thicker than $25 \mathrm{~nm}$ (solid black line). The maximally split momentum state of the pole and zero that gives rise to wideband absorption is highlighted by black dots $(11 \mathrm{~nm})$ and lies outside of this region.

adjacent metal-dielectric interfaces causes the pole and zero to acquire imaginary parts of comparable magnitude [Fig. 7(e)], especially for ultrathin films where the imaginary part of the zero becomes larger than that of the pole [Fig. 7(f)]. This strong coupling, therefore, violates condition Eq. (C5) and broadens the Lorentzian-dominated angular range Eq. (C6). Hence, even if one falsely assumes that Eq. (C3) is a valid approximation to the reflectance at those film thicknesses, the necessarily poor contrast $(b>1)$ limits the capacity of the Fano resonance term to account for pronounced features in the line shape, such as the wideband absorption of ultrathin gold films.

\section{Failure of the Fano model for ultrathin films}

From a strictly quantitative standpoint, neither the Fano line shape Eq. (C1) nor its generalized form Eq. (C3) fit the reflectance signature of gold films that are thinner than $25 \mathrm{~nm}$. This failure is shown in Fig. 10, comparing the exact reflection pole and zero wave vectors with those extracted from fitting the analytic reflectance to the generalized Fano line shape, for gold film thickness ranging from 0 to $80 \mathrm{~nm}$. The fitting is performed using the procedure described in Ref. [56], for all five coefficients $c_{1-5}$ of the generalized Fano line shape

$$
R\left(k_{z}\right)=c_{1}\left[1-\frac{c_{2}+c_{3}\left(k_{z}-c_{4}\right)}{\left(k_{z}-c_{4}\right)^{2}+c_{5}^{2}}\right],
$$

from which the fitted pole and zero wave vectors can be extracted by comparing Eqs. (C2) and (C6):

$c_{1}=D^{2}$,

$c_{2}=\left(\operatorname{Im} k_{z, \text { pole }}\right)^{2}-\left(\operatorname{Im} k_{z, \text { zero }}\right)^{2}-\left(\operatorname{Re}\left\{k_{z, \text { pole }}-k_{z, \text { zero }}\right\}\right)^{2}$,

$c_{3}=-2 \operatorname{Re}\left\{k_{z, \text { pole }}-k_{z, \text { zero }}\right\}$,

$c_{4}=\operatorname{Re} k_{z, \text { pole }}, \quad c_{5}=\operatorname{Im} k_{z, \text { pole }}$.

The exact reflection pole and zero wave vectors are attained from numerically solving Eqs. (B4) and (B5), and the analytic reflectance is calculated from the squared absolute value of the field amplitude reflection coefficient Eq. (B3), substituting the experimentally extracted film thicknesses and material permittivities, as described in Appendix B 3. The wave vector $k_{z}$ is used as the 


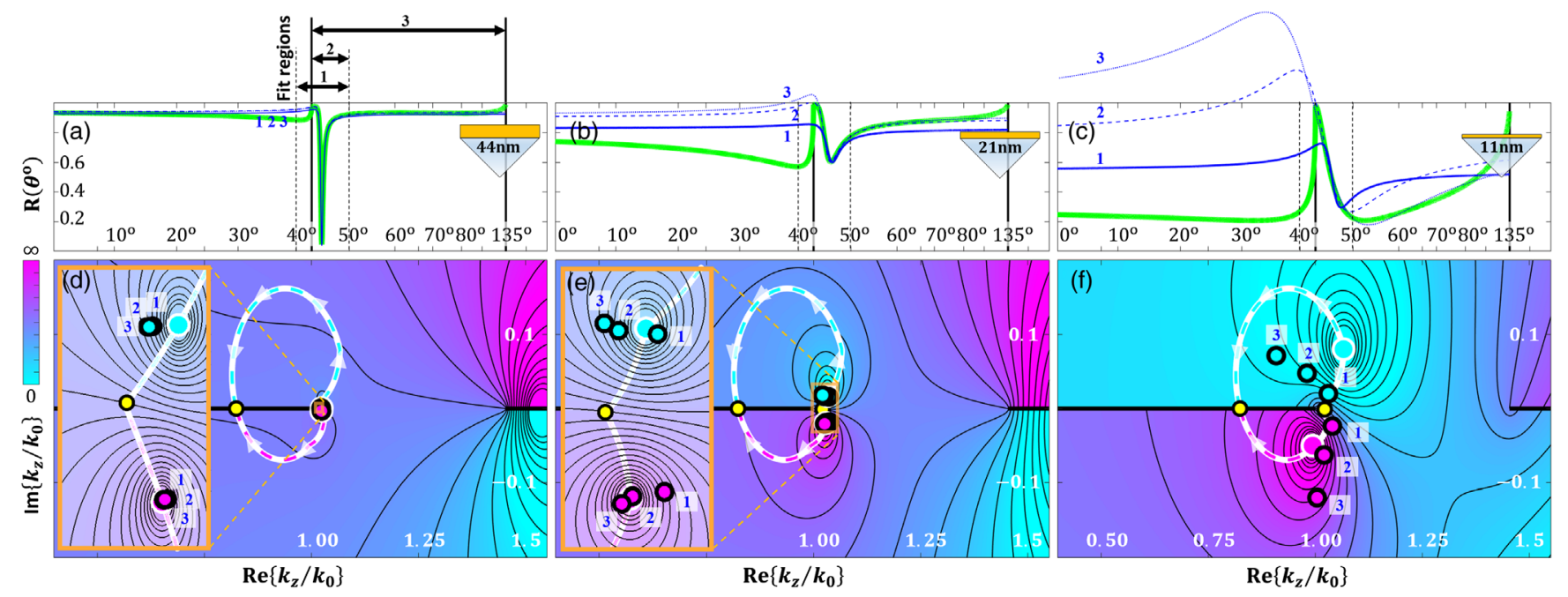

FIG. 11. Dependence of the Fano model on fitting range. Calculated reflectance of (a) 44, (B) 21 , and (c) $11 \mathrm{~nm}$ gold films (green curve) at illumination wavelength $\lambda=800 \mathrm{~nm}$, compared to the generalized Fano line shape (blue curves) fitted over three incidence angle ranges (black arrows): the range covered in our experiment (solid curve, noted 1), the subset of the former lying above the critical angle (dashed curve, noted 2), and the entire angular range accessible above the critical angle (dotted curve, noted 3). (d)-(f) The field amplitude reflection coefficient $r$ calculated over the complex wave-vector range $k_{z}$ corresponding to the angular range and film thicknesses in (a)-(c), showing the exact reflection pole (magenta) and zero (cyan) wave vectors (white circles) over their closed-loop trajectory (white curve), compared to the fitted pole and zero wave vectors (black circles) extracted from the Fano model fitted over the angular ranges $1-3$. Enlarged sections are highlighted by orange rectangles. For a genuine comparison, the reflectance $R(\theta)$ in (a)-(c) equals $\left|r\left(k_{z}\right)\right|^{2}$ and is not corrected for partial transmission at the entry and exit from the coupling prism.

independent fitting parameter, and fitting is performed over the wave-vector range that corresponds to the angular range we cover in our experiment $\left(40^{\circ} \leq \theta \leq 50^{\circ}\right)$, according to Eq. (B7) in Appendix B 6.

Figure 9 shows that the fitted pole and zero wave vectors (dotted curves) cease to track the exact values (solid curves) for gold films thinner than $25 \mathrm{~nm}$. Moreover, in that ultrathin film range, the reflectance signature cannot be fitted to the generalized Fano line shape Eq. (C3) with a reasonable coefficient of determination $\left(R^{2}>0.7\right.$, denoted by the yellow region). Consequently, the Fano model does not track the close-loop trajectory that the pole and zero follow in the wave-vector domain, most of which is traversed in the ultrathin film range. Instead, out of the wide range of wave vectors this trajectory covers, the Fano model tracks the pole and zero only across a narrow region at the vicinity of the real axis [noted by the yellow rectangle in Fig. 10(a)]. The maximally split state of the pole and zero wave vectors (11 nm, black dots), which gives rise to wideband absorption by ultrathin films [Appendix B 6 and B 9, Figs. 7(c), 7(f), and 9(b)], lies outside of this region. Therefore, the Fano model cannot reconstruct it and explain the wideband absorption we observe for ultrathin gold films.

\section{Intrinsic limitations of the Fano model}

The Fano model fails to explain the reflectance of ultrathin films because it is oversimplified and does not encompass the underlying physics. In principle, the generalized Fano line shape Eq. (C3) attempts to reduce the angular line shape to a straightforward contribution of a dominant pole and zero, resulting in a leading term attributed to a Fano resonance. However, while the angular line shape is indeed dominated by a reflection pole (SPP) and zero (APP), their contribution is not at all straightforward, because the complex wave-vector domain is topologically nontrivial (Appendix B6, Fig. 7). Hence, as shown in Fig. 11, fitting the generalized Fano line shape (blue) to the reflectance of ultrathin films (green) produces not only false values for the dispersion of the excited physical states, i.e., the pole and zero locations (black circles), but also values that strongly depend on the particular angular range over which fitting is performed (black arrows 1-3) - a clear indication that the model is underfitted and has little predictive capacity [Figs. 11(c) and 11(f)].

Beyond its inability to predict the dispersion of the excited physical states, the Fano model also qualitatively fails to track important features in the reflectance. For instance, it cannot track the shape of the narrow peak emerging just above the critical angle (solid black line at $\theta \approx 43^{\circ}$ ), especially for ultrathin gold films when it becomes pronounced [Fig. 11(c)]. This failure is because a straightforward contribution of a pole and zero cannot describe how the pole is topologically cut off from affecting the reflectance below the critical angle due to the light-line branch point, while the zero continues to affect the entire angular range (Appendix B6, Fig. 7). The former keeps the 
peak narrow, while the latter makes it pronounced on both sides of the critical angle for ultrathin films. This dynamic cannot be captured by a Fano model [Figs. 11(a)-11(c), blue curves 1-3], regardless of the angular range used for fitting (black arrows 1-3).

In principle, the Fano intensity line shape $\left[|r|^{2}\right.$, Eq. (C3)] not only identifies the amplitude transfer function of a system with a rational function having a complex pole and a complex zero [ $r$, Eq. (C2)], but also assumes these singularities project in a topologically trivial manner from the complex parameter space (wave vectors) onto its observable subspace of interest (incidence angles). When this underlying assumption is violated, such as in the case of reflectance from ultrathin gold films, even a system dominated by one pole and one zero need not obey a Fanolike behavior.

[1] C. Cohen-Tannoudji, B. Diu, and F. Laloe, Quantum Mechanics, ed. 1 (Wiley, New York, 1991), Vol. 1.

[2] D. J. Ewins, Modal Testing: Theory and Practice (Research Studies, Hertfordshire, 2000).

[3] R. E. Collin, Field Theory of Guided Waves (IEEE, New York, 1991).

[4] A. Yariv and P. Yeh, Photonics: Optical Electronics in Modern Communications, ed. 5 (Oxford, New York, 2006).

[5] E. Purcell, Spontaneous Emission Probabilities at Radio Frequencies, Phys. Rev. 69, 37 (1946).

[6] R. Piestun and D. A. B. Miller, Electromagnetic Degrees of Freedom of an Optical System, J. Opt. Soc. Am. A 17, 892 (2000).

[7] G. T. Francia, Degrees of Freedom of an Image, J. Opt. Soc. Am. 59, 799 (1969).

[8] G. Smestad, H. Ries, R. Winston, and E. Yablonovitch, The Thermodynamic Limits of Light Concentrators, Sol. Energy Mater. 21, 99 (1990).

[9] W. Chew, Waves and Fields in Inhomogeneous Media (IEEE, New York, 1995).

[10] M. F. Limonov, M. V. Rybin, A. N. Poddubny, and Y. S. Kivshar, Fano Resonances in Photonics, Nat. Photonics 11, 543 (2017).

[11] B. Luk'yanchuk, N. I. Zheludev, S. A. Maier, N. J. Halas, and P. Norlander, The Fano Resonance in Plasmonic Nanostructures and Metamaterials, Nat. Mater. 9, 707 (2010).

[12] B. Gallinet and O. J. F. Martin, Ab Initio Theory of Fano Resonances in Plasmonic Nanostructures and Metamaterials, Phys. Rev. B 83, 235427 (2011).

[13] D. Lin, P. Fan, E. Hasman, and M. L. Brongersma, Dielectric Gradient Metasurface Optical Elements, Science 345, 298 (2014).

[14] N. Yu and F. Capasso, Flat Optics with Designer Metasurfaces, Nat. Mater. 13, 139 (2014).

[15] T. Low, A. Chaves, J. D. Caldwell, A. Kumar, N. X. Fang, P. Avouris, T. F. Heinz, F. Guinea, L. Martin-Moreno, and F. Koppens, Polaritons in Layered Two-Dimensional Materials, Nat. Mater. 16, 182 (2017).
[16] D. J. Bergman and M. I. Stockman, Surface Plasmon Amplification by Stimulated Emission of Radiation: Quantum Generation of Coherent Surface Plasmons in Nanosystems, Phys. Rev. Lett. 90, 027402 (2003).

[17] M. A. Noginov, G. Zhu, A. M. Belgrave, R. Bakker, V. M. Shalaev, E. E. Narimanov, S. Stout, E. Herz, T. Suteewong, and U. Wiesner, Demonstration of a Spaser-Based Nanolaser, Nature (London) 460, 1110 (2009).

[18] R. Chikkaraddy, B. de Nijs, F. Benz, S. J. Barrow, O. A. Scherman, E. Rosta, A. Demetriadou, P. Fox, O. Hess, and J. J. Baumberg, Single-Molecule Strong Coupling at Room Temperature in Plasmonic Nanocavities, Nature (London) 535, 127 (2016).

[19] N. I. Zheludev and Y. S. Kivshar, From Metamaterials to Metadevices, Nat. Mater. 11, 917 (2012).

[20] L. Stern, B. Desiatov, I. Goykhman, and U. Levi, Nanoscale Light-Matter Interactions in Atomic Cladding Waveguides, Nat. Commun. 4, 1548 (2013).

[21] M. Brongersma, N. Halas, and P. Norlander, PlasmonInduced Hot Carrier Science and Technology, Nat. Nanotechnol. 10, 25 (2015).

[22] J. A. Schuller, E. S. Barnard, W. Cai, Y. C. Jun, J. S. White, and M. L. Brongersma, Plasmonics for Extreme Light Concentration and Manipulation, Nat. Mater. 9, 193 (2010).

[23] A. F. Koenderink, A. Alu, and A. Polman, Nanophotonics: Shrinking Light-Based Technology, Science 348, 516 (2015).

[24] M. I. Stockman et al., Roadmap on Plasmonics. J. Opt. 20, 043001 (2018).

[25] L. Novotny and B. Hecht, Principles of Nano-Optics, ed. 2 (Cambridge University, Cambridge, England, 2006).

[26] S. A. Maier, Plasmonics: Fundamentals and Applications (Springer, New York, 2007).

[27] H. Raether, Surface Plasmons on Smooth and Rough Surfaces and on Gratings (Springer, New York, 1988).

[28] F. H. L. Koppens, D. E. Chang, and F. J. G. De Abajo, Graphene Plasmonics: A Platform for Strong Light-matter Interactions, Nano. Lett. 11, 3370 (2011).

[29] A. N. Grigorenko, M. Polini, and K. S. Novoselov, Graphene Plasmonics, Nat. Photonics 6, 749 (2012).

[30] W. Cai and V. M. Shalaev, Optical Metamaterials (Springer, New York, 2010).

[31] U. Guler, A. Boltasseva, and V. M. Shalaev, Refractory Plasmonics, Science 344, 263 (2014).

[32] G. V. Naik, J. Kim, and A. Boltasseva, Oxides and Nitrides as Alternative Plasmonic Materials in the Optical Range, Opt. Mater. Express 1, 1090 (2011).

[33] A. Tsiatmas, A. R. Buckingham, V. A. Fedotov, S. Wang, Y. Chen, P. A. J. de Groot, and N. I. Zheludev, Superconducting Plasmonics and Extraordinary Transmission, Appl. Phys. Lett. 97, 111106 (2010).

[34] J. Y. Ou, J. So, G. Adamo, A. Sulaev, L. Wang, and N. I. Zheludev, Ultraviolet and Visible Range Plasmonics in the Topological Insulator $\mathrm{Bi}_{1.5} \mathrm{Sb}_{0.5} \mathrm{Te}_{1.8} \mathrm{Se}_{1.2}$, Nat. Commun. 5, 5139 (2014).

[35] I. Liberal and N. Engheta, Near-Zero Refractive Index Photonics, Nat. Photonics 11, 149 (2017).

[36] L. Novotny and N. van Hulst, Antennas for Light, Nat. Photonics 5, 83 (2011). 
[37] E. N. Economou, Surface Plasmons in Thin Films, Phys. Rev. 182, 539 (1969).

[38] G. Rosenblatt and M. Orenstein, Perfect Lensing by a Single Interface: Defying Loss and Bandwidth Limitations of Metamaterials, Phys. Rev. Lett. 115, 195504 (2015).

[39] G. Rosenblatt, G. Bartal, and M. Orenstein, Feedback as the Source of Imperfection in Lossy Perfect Lenses, Phys. Rev. A 93, 021804(R) (2016).

[40] G. Rosenblatt and M. Orenstein, Power Drainage and Energy Dissipation in Lossy but Perfect Lenses, Phys. Rev. A 95, 053857 (2017).

[41] G. Rosenblatt and M. Orenstein, Competing Coupled Gaps and Slabs for Plasmonic Metamaterial Analysis, Opt. Express 19, 20372 (2011).

[42] See Supplemental Material at http://link.aps.org/ supplemental/10.1103/PhysRevX.10.011071 for details on the origin of the tilt between the long-range SPP-APP wave vectors, and the number of antiresonance solutions in a forced system of coupled oscillators.

[43] E. Kretschmann and H. Raether, Radiative Decay of Non Radiative Surface Plasmons Excited by Light, Z. Naturforsch. A 23, 2135 (1968).

[44] J. Homola, Surface Plasmon Resonance Sensors for Detection of Chemical and Biological Species, Chem. Rev. 108, 462 (2008).

[45] P. Ferguson, R. Wallis, and M. Belakhovsky, Surface Plasma Waves in Silver and Gold, Surf. Sci. 76, 483 (1978).

[46] J. J. Burke, G. I. Stegeman, and T. Tamir, Surface-PolaritonLike Waves Guided by Thin, Lossy Metal Films, Phys. Rev. B 33, 5186 (1986).

[47] T. Wakamatsu and K. Saito, Interpretation of AttenuatedTotal-Reflection Dips Observed in Surface Plasmon Resonance, J. Opt. Soc. Am. B 24, 2307 (2007).
[48] D. Brissinger, L. Salomon, and F. De Fornel, Unguided Plasmon-Mode Resonance in Optically Excited Thin Film: Exact Modal Description of Kretschmann-Raether Experiment, J. Opt. Soc. Am. B 30, 333 (2013).

[49] Y. Akimov, M. E. Pam, and S. Sun, Kretschmann-Raether Configuration: Revision of the Theory of Resonant Interaction, Phys. Rev. B 96, 155433 (2017).

[50] D. V. Nesterenko, S. Hayashi, and Z. Sekkat, Asymmetric Surface Plasmon Resonances Revisited as Fano Resonances. Phys. Rev. B 97, 235437 (2018).

[51] A. P. Vinogradov, A. V. Dorofeenko, A. A. Pukhov, and A. A. Lisyansky, Exciting Surface Plasmon Polaritons in the Kretschmann Configuration by a Light Beam, Phys. Rev. B 97, 235407 (2018).

[52] J. J. Foley, H. Harutyunyan, D. Rosenmann, R. Divan, G. P. Wiederrecht, and S. K. Gray, When Are Surface Plasmon Polaritons Excited in the Kretschmann-Raether Configuration?, Sci. Rep. 5, 9929 (2015).

[53] J. E. Sipe, New Green-Function Formalism for Surface Optics, J. Opt. Soc. Am. B 4, 481 (1987).

[54] C. Goss, D. Charych, and M. Majda, Application of (3-Mercaptopropyl)trimethoxysilane as a Molecular Ddhesive in the Fabrication of Vapor-Deposited Gold Electrodes on Glass Substrates, Anal. Chem. 63, 85 (1991).

[55] F. Wahl, G. Schmidt, and L Forrai, On the Significance of Antiresonance Frequencies in Experimental Structural Analysis, J. Sound Vib. 219, 379 (1999).

[56] K. Kurihara, K. Nakamura, and K. Suzuki, Asymmetric SPR Sensor Response Curve-Fitting Equation for the Accurate Determination of SPR Resonance Angle, Sensors Actuators, B 86, 49 (2002). 